\title{
RIGOROUS VALIDATION OF ISOLATING BLOCKS FOR FLOWS AND THEIR CONLEY INDICES
}

By

\section{Thomas Stephens and Thomas Wanner}

\section{IMA Preprint Series \#2424}

(May 2014)

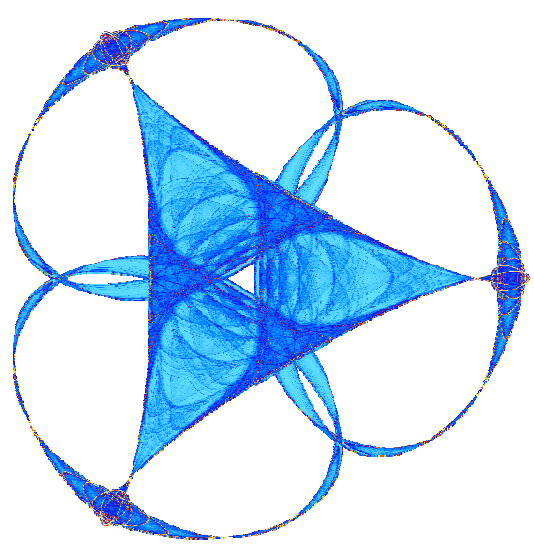

\author{
INSTITUTE FOR MATHEMATICS AND ITS APPLICATIONS \\ UNIVERSITY OF MINNESOTA \\ 400 Lind Hall \\ 207 Church Street S.E. \\ Minneapolis, Minnesota 55455-0436 \\ Phone: 612-624-6066 Fax: 612-626-7370 \\ URL: http://www.ima.umn.edu
}




\title{
RIGOROUS VALIDATION OF ISOLATING BLOCKS FOR FLOWS AND THEIR CONLEY INDICES
}

\author{
THOMAS STEPHENS* AND THOMAS WANNER*
}

\begin{abstract}
Isolated invariant sets and their associated Conley indices are valuable tools for studying dynamical systems and their global invariant structures. Through their design, they aim to capture invariant behavior which is robust under small perturbations, and this in turn makes them amenable to a computational treatment. Over the years, a number of algorithms have been proposed to find index pairs for an isolated invariant set, and then to use an index pair to compute the associated Conley index. Nevertheless, most of these methods are restricted to discrete, albeit possibly multi-valued, dynamical systems. Only relatively few general methods exist for dynamical systems generated by differential equations.

In the current paper, we present a new method for finding and rigorously verifying a special type of index pairs, namely isolating blocks and their exit sets. Our method makes use of a recently developed adaptive algorithm for rigorously determining the location of nodal sets of smooth functions, which combines an adaptive subdivision technique with interval arithmetic. By characterizing an exit set as a nodal domain, we are able to determine a valid index pair and proceed to compute its Conley index. Our method is illustrated using several examples for three-dimensional flows.
\end{abstract}

Key words. Isolated invariant set, isolating block, Conley index, computer-assisted proof, heteroclinic orbit, periodic orbit

AMS subject classifications. 37B30, 37B35, 37C10, 37C29, 37C70, 37M20

1. Introduction. In the study of ordinary differential equations and their associated flows, global invariant structures are of fundamental importance. In contrast to the case of stationary or equilibrium points, these structures consist of non-constant bounded solutions of the underlying system, such as periodic, homoclinic, or heteroclinic solutions, or even more complicated objects such as strange attractors. They can be representations of periodic or other recurrent behavior, or they can delineate between regions of phase space with different asymptotic behavior, for example as boundaries of domains of attraction. Many applications, such as the study of traveling waves in partial differential equations, naturally lead to the necessity of finding global invariant structures in ordinary differential equations. While local analysis certainly plays an important role in this task, it alone cannot suffice.

Over the years, a number of tools have been developed to aid in locating global invariant structures and rigorously prove their existence, most of them topological in nature. One of the most flexible and powerful among these is Conley's theory of the homotopy index $[4,18,21]$. Since this theory is the foundation for our approach, we begin by briefly recalling its basics. In the following, we consider a smooth ordinary differential equation of the form

$$
\dot{x}=f(x)
$$

and assume that it generates a smooth global flow $\varphi: \mathbb{R} \times \mathbb{R}^{n} \rightarrow \mathbb{R}^{n}$. A set $\mathcal{S} \subset \mathbb{R}^{n}$ in the phase space is called invariant under the flow $\varphi$ if we have $\varphi(\mathbb{R}, \mathcal{S}) \subset \mathcal{S}$, i.e., if for every point $x \in \mathcal{S}$ the full orbit through $x$ stays in $\mathcal{S}$. The smallest sets in phase space which are invariant under $\varphi$ are of course single orbits, as well as arbitrary unions of orbits, but one is generally interested in invariant sets with some additional structure or desirable property — most notably boundedness of $\mathcal{S}$. Many invariant

*Department of Mathematical Sciences, George Mason University, Fairfax, VA 22030, USA 
sets that capture interesting dynamics are in fact bounded invariant sets, for example fixed points, periodic orbits, and connecting orbits.

As important as bounded invariant sets are for understanding a particular dynamical system, they are often equally difficult to resolve computationally. Ordinary differential equations such as (1.1) which arise in applications generally involve parameters in the definition of the right-hand side $f$. Bounded invariant sets, however, can change dramatically with changes in the parameter, and this sensitivity can have profound impact on their computability - even if the right-hand side $f$ is known exactly. Furthermore, the parameters affecting $f$ are often not known precisely, and one is therefore interested in uncovering bounded invariant sets which exist in similar form for whole ranges of parameter values. Finally, in many systems the most important bounded invariant sets act as organizing centers for the dynamics, and are therefore themselves unstable, making direct numerical approximation extremely difficult. Based on these comments, it is not surprising that any theoretical approach to the study of bounded invariant sets which is useful for applications should in some sense be robust to perturbations of the right-hand side $f$ in (1.1).

One such approach was proposed by Conley [4], who realized that robustness with respect to continutation can be achieved by restricting attention to special invariant sets. Let $\mathcal{N} \subset \mathbb{R}^{n}$ denote any compact set. Then the maximal invariant set in $\mathcal{N}$ is defined by

$$
\operatorname{Inv}(\mathcal{N})=\{x \in \mathcal{N}: \varphi(\mathbb{R}, x) \subset \mathcal{N}\}
$$

This definition assigns a possibly empty invariant set to every compact subset of phase space. The basis for Conley theory are those which do not touch the boundary of $\mathcal{N}$. More precisely, an invariant set $\mathcal{S}$ is called an isolated invariant set, if there exists a compact subset $\mathcal{N} \subset \mathbb{R}^{n}$ such that

$$
\mathcal{S}=\operatorname{Inv}(\mathcal{N}) \subset \operatorname{int} \mathcal{N}
$$

where int $\mathcal{N}$ denotes the interior of $\mathcal{N}$. In this case, the set $\mathcal{N}$ is called an isolating neighborhood. In addition to being the maximal invariant set in a compact neighborhood $\mathcal{N}$, an isolated invariant set $\mathcal{S}$ also cannot touch the boundary of $\mathcal{N}$. Together, these properties imply that if a compact set $\mathcal{N}$ is an isolating neighborhood for an ordinary differential equation (1.1), then it is also an isolating neighborhood for $\dot{x}=g(x)$, if the new right-hand side $g$ is a sufficiently small perturbation of $f$. In other words, even though small changes in $f$ can change $\mathcal{S}=\operatorname{Inv}(\mathcal{N})$, the compact set $\mathcal{N}$ itself still remains an isolating neighborhood, and therefore uniquely determines an isolated invariant set in its interior. For more details, see [4, 18, 21].

In [4], Conley showed that it is possible to define an index theory for isolating neighborhoods $\mathcal{N}$, which in many ways mimics the Brouwer degree for continuous mappings. In particular, this index theory has the Wazewski property: If the index is non-trivial, then the isolated invariant set $\mathcal{S}=\operatorname{Inv}(\mathcal{N})$ is non-empty. Moreover, if $\mathcal{N}_{1}$ and $\mathcal{N}_{2}$ are two isolating neighborhoods for the same isolated invariant set $\mathcal{S}$, then the indices assigned to $\mathcal{N}_{1}$ and $\mathcal{N}_{2}$ coincide. In other words, the Conley index of $\mathcal{N}$ is actually an index for the isolated invariant set $\mathcal{S}=\operatorname{Inv}(\mathcal{N})$.

Conley's index theory recognizes that the flow which is not part of the isolated invariant set $\mathcal{S}$, yet is contained in an isolating neighborhood for $\mathcal{S}$, shuttles along its topology via a homotopy. Thus, computations away from the isolated invariant set have a chance of revealing information about the invariant set itself. The classical definition of the Conley index is a reflection of this idea, and is based on the notion of 
index pair. Assume that $\mathcal{S}$ is an isolated invariant set for a flow $\varphi$. A pair of compact sets $(\mathcal{N}, \mathcal{L})$ with $\mathcal{L} \subset \mathcal{N}$ is called an index pair for $\mathcal{S}$, if the following hold:

- $\mathcal{N} \backslash \mathcal{L}$ is a neighborhood of $\mathcal{S}$, and $\mathcal{S}=\operatorname{Inv}(\overline{\mathcal{N} \backslash \mathcal{L}})$.

- $\mathcal{L}$ is positively invariant in $\mathcal{N}$ : If $x \in \mathcal{L}$, then the orbit $\varphi(t, x)$ for $t \geq 0$ stays in $\mathcal{L}$ as long as it stays in $\mathcal{N}$.

- $\mathcal{L}$ is an exit set for $\mathcal{N}$ : If the orbit starting at $x \in \mathcal{N}$ exits $\mathcal{N}$ in forward time, it has to enter $\mathcal{L}$ before it exits $\mathcal{N}$.

One can show that for every isolated invariant set $\mathcal{S}$ there do exist many index pairs. Given any such index pair, the homotopy Conley index of $\mathcal{S}$ is defined as the pointed space

$$
h(\mathcal{S})=(\mathcal{N} / \mathcal{L},[\mathcal{L}])
$$

and the weaker, but often sufficient, homological Conley index of $\mathcal{S}$ is defined as

$$
C H_{*}(\mathcal{S})=H_{*}(\mathcal{N} / \mathcal{L},[\mathcal{L}]) \approx H_{*}(\mathcal{N}, \mathcal{L})
$$

Using the idea of flow transport mentioned above, one can show that the Conley index in either form is independent (up to isomorphism) of the choice of index pair, and only depends on the underlying isolated invariant set $\mathcal{S}$. Note, however, that for the computation of the Conley index, only the index pair is necessary. For the purposes of this paper, we will always consider the homological Conley index which is given by a sequence $\left(\mathrm{CH}_{k}(\mathcal{S})\right)_{k=0}^{\infty}$ of Abelian groups $\mathrm{CH}_{k}(\mathcal{S})$. The Ważewski principle can then be stated as follows: If at least one of the homology groups $C H_{k}(\mathcal{S})$ is non-trivial, then $\mathcal{S} \neq \emptyset$.

The main goal of the present work is to develop a computational techique for rigorously computing the Conley index of an isolated invariant set. Rather than considering general index pairs, we focus on the following special case which was introduced in [5], see also [18].

Definition 1.1. A compact set $\mathcal{B} \subset \mathbb{R}^{n}$ is called an isolating block for the flow $\varphi$ generated by the ordinary differential equation (1.1), if its exit set

$$
\mathcal{B}^{-}=\{x \in \mathcal{B}: \varphi([0, T], x) \not \subset \mathcal{B} \text { for all } T>0\}
$$

is closed, and if for all $T>0$ we have

$$
\operatorname{Inv}_{T}(\mathcal{B}, \varphi)=\{x \in \mathcal{B}: \varphi([-T, T], x) \subset \mathcal{B}\} \subset \operatorname{int} \mathcal{B} .
$$

Note that the closedness of the exit set $\mathcal{B}^{-}$is part of the assumption of an isolating block, and that it does not follow automatically from (1.5).

It was shown in [5] that for every isolated invariant set there exist associated isolating blocks $\mathcal{B}$. Given an isolating block $\mathcal{B}$ as above, in general some trajectories of the flow which originate in $\mathcal{B}$ will leave $\mathcal{B}$. If they do, they have to pass through the exit set $\mathcal{B}^{-}$as defined in (1.4). This set consists of points that are immediately pushed out of $\mathcal{B}$ in forward time. Moreover, if we define

$$
\mathcal{B}^{+}=\{x \in \mathcal{B}: \varphi([-T, 0], x) \not \subset \mathcal{B} \text { for all } T>0\}
$$

then one can easily see that (1.5) is equivalent to the identity

$$
\partial \mathcal{B}=\mathcal{B}^{-} \cup \mathcal{B}^{+} .
$$


This implies two things. First of all, the invariant set $\operatorname{Inv}(\mathcal{B})$ has to be contained in the interior of $\mathcal{B}$, i.e., $\mathcal{B}$ is an isolating neighborhood. Secondly, any orbit which originates in $\mathcal{B}$ and which touches the boundary $\partial \mathcal{B}$ has to immediately exit the isolating block either forward or backward in time. In other words, the flow does not generate any internal tangencies with the boundary of $\mathcal{B}$. Thus, one can easily see that the following holds.

LEMMA 1.2. If the compact set $\mathcal{B} \subset \mathbb{R}^{n}$ is an isolating block for the flow $\varphi$ generated by the ordinary differential equation (1.1), and if $\mathcal{S}=\operatorname{Inv}(\mathcal{B})$ denotes the associated isolated invariant set, then the pair $\left(\mathcal{B}, \mathcal{B}^{-}\right)$is an index pair for $\mathcal{S}$.

Index pairs are the fundamental objects in Conley's theory, since their knowledge alone enables one to determine the Conley index. This in turn furnishes the Conley index of the associated, but usually unknown, isolated invariant set $\mathcal{S}$. It is therefore not surprising that many algorithmic approaches have been developed over the years to construct index pairs computationally. Most of these have been derived for discretetime dynamical systems, or for the case of multi-valued maps. For more information, we refer the reader to $[15,18]$ and the references therein. Finding index pairs for flows turns out to be a much more complicated task. Many approaches reduce the problem to the discrete-time setting by constructing rigorous outer approximations of a time- $T$-map for the flow, which of course involves approximating the solutions of the ordinary differential equation (1.1).

Methods which avoid the above-mentioned time integration and work directly with the vector field $f$ are rarer. We briefly describe two of them in more detail, since they are most directly related to our work. The first approach is due to Boczko, Kalies, and Mischaikow [1, 2], and it is aimed at decomposing the underlying finitedimensional phase space into a simplicial complex which can be used to track the flow induced by (1.1). More precisely, every simplex in the constructed complex is aligned with the flow in such a way that along each of the faces of the simplex the flow is transversal. Thus, one can create a multi-valued map which describes the possible paths of orbits and which can be used to find index pairs for the flow. However, while constructing a suitable simplicial decomposition is feasible in two and three dimensions, it becomes considerably more complicated in higher dimensions. Another approach is due to Eberlein and Scheurle [11, 12]. Rather than constructing a flowinduced decomposition of phase space, their work is concentrating on the numerical construction of specific isolating blocks for flows. For polyhedral regions in two and three dimensions, they use Delaunay triangulations to try to resolve possible internal flow tangencies and arrive at a modified polyhedral region which can then be used to form an isolating block. The associated homological Conley index is computed using the Delaunay triangulation, in combination with a variety of contractibility conditions and the nerve lemma. However, even in the considered two and three-dimensional examples, resolving the internal tangencies, as well as an a-posteriori rigorous computational validation of the isolating block using interval arithmetic seems extremely involved. Furthermore, the approach in $[11,12]$ is very much relying on the fact that the faces of the polyhedral regions are flat, and they did not address resolving internal tangencies in dimensions higher than three.

In the current paper we present a method which allows for the automated rigorous verification of isolating blocks $\mathcal{B} \subset \mathbb{R}^{n}$ for (1.1) whose boundary is a smooth orientable compact manifold $\mathcal{M}$ in $\mathbb{R}^{n}$ of codimension one. In this sense, our approach is similar in spirit to $[11,12]$, even though it no longer requires flat polygons to bound the region. However, our approach differs significantly in that we derive an 
easily stated condition which can readily be verified using rigorous computations based on interval arithmetic, and which either provides a computer-assisted proof that the given region is an isolating block, or provides guidance as to where the manifold $\mathcal{M}$ has to be adjusted in order to arrive at an isolating block. Our condition is based on properties of nodal domains and nodal sets of smooth functions, and applies in arbitrary dimensions $n$. Recall that if $u: \mathcal{M} \rightarrow \mathbb{R}$ is any real-valued function, then its positive and negative nodal domains $N^{ \pm}(u)$ are defined as the sets

$$
N^{+}(u)=\{p \in \mathcal{M}: u(p)>0\} \quad \text { and } \quad N^{-}(u)=\{p \in \mathcal{M}: u(p)<0\},
$$

and its nodal set $N^{0}(u)$ is defined by

$$
N^{0}(u)=\{p \in \mathcal{M}: u(p)=0\}
$$

While at this point we do not want to present all the details, our main theorem takes the following form. For the precise formulation, see Theorem 2.3 below.

Theorem 1.3 (Isolating Blocks via Nodal Domains). Consider the flow $\varphi$ associated with the ordinary differential equation (1.1), and assume that the boundary of the compact set $\mathcal{B} \subset \mathbb{R}^{n}$ is given by a smooth orientable compact embedded manifold $\mathcal{M}$ of codimension one. Then there exist smooth functions $u, v: \mathcal{M} \rightarrow \mathbb{R}$ which depend only on $\mathcal{M}$ and $f$ such that the following holds. If we have

$$
N^{0}(u) \subset N^{+}(v), \quad \text { and if } z \text { ero is a regular value of } u \text {, }
$$

then $\mathcal{B}$ is an isolating block for (1.1) in the sense of Definition 1.1. In this case, the exit set $\mathcal{B}^{-}$defined in (1.4) is given by the closure of the positive nodal domain $N^{+}(u)$ of $u$, i.e., we have $\mathcal{B}^{-}=\overline{N^{+}(u)}$. The definition of the functions $u$ and $v$ can be found in (2.4) and (2.5) below.

By reformulating the test for an isolating block in terms of nodal domains of functions, we can make use of a recent algorithm $[3,6,7]$ which rigorously validates such nodal domains using interval arithmetic and an adaptive randomized subdivision procedure. The essential aspects of this algorithm will be recalled later, but it can easily be implemented in Matlab using for example the interval arithmetic package INTLAB [20], and an implementation in C++ for arbitrary dimensions has recently been completed [9]. The output of either algorithm is a nonuniform rectangular grid, from which the Conley index associated with the isolating block can be computed using the technique developed in [8].

The remainder of this paper is organized as follows. In Section 2 we present the precise definitions of the two test functions $u$ and $v$ from Theorem 1.3 and establish the result. The next section is completely devoted to the computational aspects involved in applying Theorem 1.3 in practice. In Section 3.1 we recall the algorithm of $[3,6,7]$ to validate nodal domains, and in Sections 3.2 and 3.3 we describe the extensions which are necessary in our situation. While the bulk of our paper is concerned with regions which are bounded by a smooth manifold, in Section 3.4 we address the problem of regions whose boundary is a piece-wise smooth manifold. Finally, in Section 4 we present three case studies to illustrate our method. In Section 4.1 we discuss a gradient flow example from [11], followed by an example of a non-gradient flow in Section 4.2 which is taken from [2]. The paper concludes with a Hopf bifurcation example from [12] in Section 4.3. 
2. Characterization of Isolating Blocks via Nodal Domains. In this section we provide the theoretical background for our method by establishing the characterization of isolating blocks which was briefly sketched in Theorem 1.3. Throughout this section, we make the following assumptions and notational definitions.

(A1) We assume that $\mathcal{B} \subset \mathbb{R}^{n}$ is a connected open subset whose boundary $\mathcal{M}=\partial \mathcal{B}$ is a smooth compact embedded orientable manifold in $\mathbb{R}^{n}$ of codimension one. In this case, the manifold $\mathcal{M}$ divides the ambient space $\mathbb{R}^{n}$ into the bounded component $\mathcal{B}$ and the unbounded component $\mathbb{R}^{n} \backslash \overline{\mathcal{B}}$, see for example [13, Theorem 4.6]. The normal and tangential directions to the manifold are defined as follows.

(A2) For every $p \in \mathcal{M}$ we denote by $T_{p} \mathcal{M} \subset \mathbb{R}^{n}$ the tangent space of $\mathcal{M}$ at $p$. Furthermore, we assume that $\mathcal{M} \ni p \mapsto \nu_{p} \in \mathbb{R}^{n}$ is a smooth mapping with the property that $\nu_{p} \neq 0$ is an outward normal vector for $\mathcal{B}$ for every $p \in \mathcal{M}$. Finally, for every vector $q \in \mathbb{R}^{n}$ we denote its orthogonal projection onto $T_{p} \mathcal{M}$ by $\pi_{p}^{\top} q$.

In our setting, the tangent spaces $T_{p} \mathcal{M}$ are of codimension one, and standard results on orientable manifolds imply that smooth normal vector mappings $\nu: \mathcal{M} \rightarrow \mathbb{R}^{n}$ as in (A2) always exist. In fact, if we require in addition that the normal vectors are all normalized to length one, then this vector field is uniquely determined. See for example [17, Proposition 13.21, Problem 15-7]. Note, however, that in (A2) we do not require any normalization of $\nu_{p}$ in order to keep our approach as flexible as possible. The projection operator $\pi_{p}^{\top}$ is explicitly given by

$$
\pi_{p}^{\top} q=q-\frac{\left\langle q, \nu_{p}\right\rangle}{\left\langle\nu_{p}, \nu_{p}\right\rangle} \cdot \nu_{p},
$$

where $\langle\cdot, \cdot\rangle$ denotes the standard scalar product on $\mathbb{R}^{n}$. Finally, we also need to make use of the following notion from Riemannian geometry.

(A3) For every $p \in \mathcal{M}$ we denote by $\mathbb{I}: T_{p} \mathcal{M} \times T_{p} \mathcal{M} \rightarrow \mathbb{R}$ the second fundamental form of $\mathcal{M}$ at $p$ along the normal vector field $\nu$.

The second fundamental form measures curvature information of the manifold $\mathcal{M}$ and will prove to be useful for excluding internal tangencies of the flow. For more details we refer the reader to $[10,16]$.

After these preparations we can now turn our attention to the definition of the functions $u$ and $v$ mentioned in Theorem 1.3. This will be separated into two lemmas, the first of which addresses the question of domain exit from $\mathcal{B}$.

LEMMA 2.1. Consider the ordinary differential equation (1.1) with smooth righthand side $f$, and suppose that Assumptions (A1) and (A2) are satisfied. Furthermore, define the function $u: \mathcal{M} \rightarrow \mathbb{R}$ as

$$
u(p)=\left\langle f(p), \nu_{p}\right\rangle,
$$

where $\nu$ denotes the normal vector field from (A2). Then we have the inclusions

$$
N^{+}(u) \subset \mathcal{B}^{-} \backslash \mathcal{B}^{+} \quad \text { and } \quad N^{-}(u) \subset \mathcal{B}^{+} \backslash \mathcal{B}^{-},
$$

where $N^{ \pm}(u)$ were defined in (1.8), and $\mathcal{B}^{ \pm}$where defined in (1.4) and (1.6). Notice that at this point we cannot make any statements about the behavior of the flow near points $p \in \mathcal{M}$ with $u(p)=0$.

Proof. Let $p \in \mathcal{M}$. According to [17, Proposition 5.16], there exists a neighborhood $U \subset \mathbb{R}^{n}$ of $p$ and a smooth submersion $\Phi: U \rightarrow \mathbb{R}$ such that $\mathcal{M} \cap U$ is given by the zero set of $\Phi$. Since $\nabla \Phi(p)$ is orthogonal to $T_{p} \mathcal{M}$, we can assume without loss of 
generality that $\nabla \Phi(p)$ is a positive multiple of $\nu_{p}$. Thus, for all $q \in U$ we have $q \notin \mathcal{B}$ if and only if $\Phi(q)>0$, and $q$ is contained in the interior of $\mathcal{B}$ if and only if $\Phi(q)<0$.

Now let $x(t)$ denote the solution of (1.1) satisfying $x(0)=p$. Evaluating $\Phi$ along the solution $x(t)$ close to $p$ and differentiating with respect to $t$ then furnishes

$$
\frac{d}{d t} \Phi(x(t))=\langle\nabla \Phi(x(t)), \dot{x}(t)\rangle=\langle\nabla \Phi(x(t)), f(x(t))\rangle .
$$

For $t=0$ one therefore has

$$
\left.\frac{d}{d t} \Phi(x(t))\right|_{t=0}=\langle\nabla \Phi(p), f(p)\rangle=c \cdot u(p),
$$

where $c>0$ is a positive scalar. If we now assume $u(p)>0$, then there exists a $T>0$ such that $\Phi(x(t))>0$ for all $t \in(0, T]$, and $\Phi(x(t))<0$ for all $t \in[-T, 0)$. Together with the above characterization of the interior and exterior of $\mathcal{B}$ in $U$ given above, this immediately implies (a). Since the proof of $(b)$ is completely analogous, Lemma 2.1 follows.

Notice that the above lemma only uses the normal vector field $\nu$ on the manifold $\mathcal{M}=\partial \mathcal{B}$, and it allows us to locate the exit set $\mathcal{B}^{-}$up to points at which $u(p)=0$. These points of course are candidates for flow tangencies with the boundary of $\mathcal{B}$. In order to exclude internal tangencies, understanding the local curvature of the manifold is crucial. Thus, the following lemma, which introduces the tangency test function $v$ mentioned in Theorem 1.3, needs to make use of the second fundamental form of the manifold $\mathcal{M}$.

LEMMA 2.2. Consider the ordinary differential equation (1.1) with smooth righthand side $f$, and suppose that Assumptions (A1) through (A3) are satisfied. Let $u$ denote the function from (2.1), and define the function $v: \mathcal{M} \rightarrow \mathbb{R}$ as

$$
v(p)=\left\langle D f(p) f(p), \nu_{p}\right\rangle-\mathbb{I}\left(\pi_{p}^{\top} f(p), \pi_{p}^{\top} f(p)\right),
$$

where $\nu$ denotes the normal vector field from (A2) and II the second fundamental form from (A3). If the point $p \in \mathcal{M}=\partial \mathcal{B}$ satisfies $u(p)=0$ and $v(p)>0$, then the solution of (1.1) through $p$ forms an external tangency with $\partial \mathcal{B}$, i.e., there exists a time $T>0$ such that $\varphi([-T, T], p) \cap \mathcal{B}=\{p\}$, where $\varphi$ denotes the flow associated with (1.1). On the other hand, if $u(p)=0$ and $v(p)<0$, then the solution of (1.1) through $p$ forms an internal tangency with $\partial \mathcal{B}$, i.e., there exists a time $T>0$ such that $\varphi([-T, T], p) \backslash\{p\} \subset$ int $\mathcal{B}$.

Proof. Let $p \in \mathcal{M}$. As in the proof of the previous lemma, there exists a neighborhood $U \subset \mathbb{R}^{n}$ of $p$ and a smooth submersion $\Phi: U \rightarrow \mathbb{R}$ such that $\mathcal{M} \cap U$ is given by the zero set of $\Phi$, and that $\nabla \Phi(p)$ is a positive multiple of $\nu_{p}$. Now let $\alpha: U \rightarrow \mathbb{R}^{+}$ be a smooth function with $\alpha(q)=\left\|\nu_{q}\right\|$ for all $q \in \mathcal{M} \cap U$. Such a function can always be constructed, possibly after choosing a smaller neighborhood $U$. For example, using a tubular neighborhood of $\mathcal{M} \cap U$ as in [13, Theorem 5.1], one can simply define $\alpha$ to be constant on each of the normal fibres. If we then define $\Psi: U \rightarrow \mathbb{R}$ via $\Psi(q)=\alpha(q) \Phi(q) /\|\nabla \Phi(q)\|$ for all $q \in U$, then also $\Psi$ is a smooth submersion such that $\mathcal{M} \cap U$ is given by the zero set of $\Psi$. In addition, one can easily see that

$$
\nabla \Psi(q)=\nu_{q} \quad \text { for all } \quad q \in \mathcal{M} \cap U .
$$

Thus, we still have for all $q \in U$ that $q \notin \mathcal{B}$ if and only if $\Psi(q)>0$, and $q$ is contained in the interior of $\mathcal{B}$ if and only if $\Psi(q)<0$. But in addition, the smooth vector field $\nabla \Psi$ is an extension of $\nu$. onto $U$. 
After these preparations, let $p \in \mathcal{M}$ be a point with $u(p)=0$, and let $x(t)$ denote the solution of (1.1) with $x(0)=p$. If we define the real-valued function $\tau$ in a small neighborhood of $0 \in \mathbb{R}$ via $\tau(t)=\Psi(x(t))$, then $\tau(0)=\Psi(p)=0$. Furthermore, due to the above construction of $\Psi$, the solution $x(t)$ forms an external tangency with $\partial \mathcal{B}=\mathcal{M}$ if there exists a time $T>0$ such that $\tau(t)>0$ for all $t \in[-T, T] \backslash\{0\}$. Similarly, $x(t)$ forms an internal tangency if we have $\tau(t)<0$ on $[-T, T] \backslash\{0\}$. As in the proof of Lemma 2.1, the derivative of $\tau$ can be computed as

$$
\tau^{\prime}(t)=\frac{d}{d t} \Psi(x(t))=\langle\nabla \Psi(x(t)), \dot{x}(t)\rangle=\langle\nabla \Psi(x(t)), f(x(t))\rangle,
$$

which implies $\tau^{\prime}(0)=\langle\nabla \Psi(p), f(p)\rangle=\left\langle\nu_{p}, f(p)\right\rangle=u(p)=0$ due to (2.2). The second derivative of $\tau$ is given by

$$
\tau^{\prime \prime}(t)=\langle\nabla \Psi(x(t)), D f(x(t)) f(x(t))\rangle+\langle\operatorname{Hess} \Psi(x(t)) f(x(t)), f(x(t))\rangle,
$$

and evaluation at $t=0$ furnishes in combination with (2.2) the identity

$$
\tau^{\prime \prime}(0)=\left\langle\nu_{p}, D f(p) f(p)\right\rangle+\langle\operatorname{Hess} \Psi(p) f(p), f(p)\rangle .
$$

According to our above discussion, the solution $x(t)$ forms an external tangency with $\partial \mathcal{B}=\mathcal{M}$ if $\tau^{\prime \prime}(0)>0$, and an internal tangency if $\tau^{\prime \prime}(0)<0$. Thus, in order to complete the proof of the lemma it suffices to show that $\tau^{\prime \prime}(0)=v(p)$. Since we already know that $f(p) \in T_{p} \mathcal{M}$ (recall that $u(p)=0$ ), this in turn follows immediately from the fact that

$$
\mathbb{I}(y, z)=-\langle\operatorname{Hess} \Psi(p) y, z\rangle \quad \text { for all } \quad y, z \in T_{p} \mathcal{M},
$$

which will be established in the remainder of the proof.

In order to verify (2.3), we make use of some results from Riemannian geometry. According to [10, Chapter 6, Proposition 2.3], the second fundamental form is given by

$$
\mathbb{I}(y, z)=-\left\langle\pi_{p}^{\top} \bar{\nabla}_{y} \nabla \Psi(p), z\right\rangle \quad \text { for all } \quad y, z \in T_{p} \mathcal{M} .
$$

In this formula, $\pi_{p}^{\top}$ denotes the orthogonal projection onto $T_{p} \mathcal{M}$ introduced in (A2), and $\bar{\nabla}$ denotes the Riemannian connection on $\mathbb{R}^{n}$. For any vector field $N$ on $\mathbb{R}^{n}$, the latter is given by the directional derivative $\bar{\nabla}_{y} N=D N y$, see $[10$, pp. 51-56]. This yields

$$
\pi_{p}^{\top} \bar{\nabla}_{y} \nabla \Psi(p)=\pi_{p}^{\top} \operatorname{Hess} \Psi(p) y=\operatorname{Hess} \Psi(p) y-\frac{\left\langle\operatorname{Hess} \Psi(p) y, \nu_{p}\right\rangle}{\left\langle\nu_{p}, \nu_{p}\right\rangle} \cdot \nu_{p},
$$

as well as $\mathbb{I}(y, z)=-\langle\operatorname{Hess} \Psi(p) y, z\rangle$, since $z \in T_{p} \mathcal{M}$ implies $\left\langle\nu_{p}, z\right\rangle=0$. This completes the verification of (2.3), and thus also of Lemma 2.2.

After these preparations, we can now proceed to the characterization of isolating blocks via nodal sets and nodal domains as described briefly in the introduction. For the convenience of the reader, we restate Theorem 1.3 before proving it, this time including all the necessary details.

TheOREM 2.3 (Isolating Blocks via Nodal Domains). Consider the flow $\varphi$ associated with the ordinary differential equation (1.1), and suppose that Assumptions (A1) through (A3) are satisfied, i.e., assume that the boundary of the compact set $\mathcal{B} \subset \mathbb{R}^{n}$ 
is given by a smooth compact embedded orientable manifold $\mathcal{M}$ of codimension one. Furthermore, define the smooth functions $u, v: \mathcal{M} \rightarrow \mathbb{R}$ via

$$
\begin{aligned}
& u(p)=\left\langle f(p), \nu_{p}\right\rangle, \\
& v(p)=\left\langle D f(p) f(p), \nu_{p}\right\rangle-\mathbb{I}\left(\pi_{p}^{\top} f(p), \pi_{p}^{\top} f(p)\right) .
\end{aligned}
$$

If in addition we have

$$
N^{0}(u) \subset N^{+}(v), \quad \text { and if zero is a regular value of } u,
$$

then $\mathcal{B}$ is an isolating block for (1.1) in the sense of Definition 1.1. In this case, the exit set $\mathcal{B}^{-}$defined in (1.4) is given by

$$
\mathcal{B}^{-}=N^{+}(u) \cup N^{0}(u)=\overline{N^{+}(u)},
$$

i.e., by the closure of the positive nodal domain $N^{+}(u)$ of $u$.

Proof. According to the definition of the nodal domains and the nodal set in (1.8) and (1.9), respectively, the boundary of $\mathcal{B}$ can be partitioned in the form

$$
\mathcal{M}=N^{+}(u) \cup N^{0}(u) \cup N^{-}(u) .
$$

Due to Lemma 2.1 we have both $N^{+}(u) \subset \mathcal{B}^{-} \backslash \mathcal{B}^{+}$and $N^{-}(u) \subset \mathcal{B}^{+} \backslash \mathcal{B}^{-}$. Now let $p \in N^{0}(u)$ be arbitrary. Then (2.6) implies $p \in N^{+}(v)$, and Lemma 2.2 shows that the solution of (1.1) through $p$ forms an external tangency with $\mathcal{B}$, i.e., there exists a $T>0$ such that $\varphi([-T, T], p) \cap \mathcal{B}=\{p\}$, where $\varphi$ denotes the flow associated with (1.1). This readily implies $p \in \mathcal{B}^{-} \cap \mathcal{B}^{+}$, i.e., we have $N^{0}(u) \subset \mathcal{B}^{-} \cap \mathcal{B}^{+}$. As mentioned above, the sets $N^{ \pm}(u)$ and $N^{0}(u)$ form a partition of $\mathcal{M}$. Since in addition the sets $\mathcal{B}^{-} \backslash \mathcal{B}^{+}, \mathcal{B}^{+} \backslash \mathcal{B}^{-}$, and $\mathcal{B}^{-} \cap \mathcal{B}^{+}$are pairwise disjoint, we obtain both

$$
\partial \mathcal{B}=\left(\mathcal{B}^{-} \backslash \mathcal{B}^{+}\right) \cup\left(\mathcal{B}^{+} \backslash \mathcal{B}^{-}\right) \cup\left(\mathcal{B}^{-} \cap \mathcal{B}^{+}\right),
$$

as well as

$$
N^{+}(u)=\mathcal{B}^{-} \backslash \mathcal{B}^{+}, \quad N^{-}(u)=\mathcal{B}^{+} \backslash \mathcal{B}^{-}, \quad \text { and } \quad N^{0}(u)=\mathcal{B}^{-} \cap \mathcal{B}^{+} .
$$

The first statement shows that (1.5) in Definition 1.1 is satisfied. Moreover, due to the second statement we have $\mathcal{B}^{-}=N^{+}(u) \cup N^{0}(u)$, and thus $\mathcal{B}^{-}=\{p \in \mathcal{M}: u(p) \geq 0\}$. Due to the continuity of $u$ this set is closed. Together, this implies that the set $\mathcal{B}$ is an isolating block in the sense of Definition 1.1.

In order to complete the proof we still need to verify that $\mathcal{B}^{-}=\overline{N^{+}(u)}$. Due to $N^{+}(u) \subset \mathcal{B}^{-}$and the closedness of $\mathcal{B}^{-}$one clearly has $\overline{N^{+}(u)} \subset \mathcal{B}^{-}$. Since we have already established $\mathcal{B}^{-}=N^{+}(u) \cup N^{0}(u)$, we only need to show that $N^{0}(u) \subset \overline{N^{+}(u)}$. According to [17, Corollary 5.14], the nodal set $N^{0}(u) \subset \mathcal{M}$ is a properly embedded submanifold of codimension one, since we assumed that zero is a regular value of $u$. Now let $p \in N^{0}(u)$ be arbitrary. Since $u$ is a submersion at $p$, every neighborhood of $p$ in $\mathcal{M}$ has to contain points which lie in $N^{+}(u)$, and points in $N^{-}(u)$. This readily shows that $p \in \overline{N^{+}(u)}$, and the proof of Theorem 2.3 is complete. $\square$

We would like to point out that in the above theorem, the set $\mathcal{B}$ is an isolating block even if zero is not a regular value of $u$. However, in this case one cannot in general show that the exit set $\mathcal{B}^{-}$is the closure of the positive nodal domain $N^{+}(u)$. This technicality will not affect our further studies, since the validation algorithm which will be discussed in the next section automatically implies that zero is a regular 
value. In fact, we will see that in principle the algorithm cannot succeed if zero is not a regular value of $u$ - and a new candidate $\mathcal{B}$ would have to be chosen.

To close this section, we collect a couple of formulas which are hidden in the proofs of Lemmas 2.1 and 2.2, but deserve to be stated explicitly. Assume that the manifold $\mathcal{M}$ in Theorem 2.3 is given as the nodal set of a smooth function $\Phi: \mathbb{R}^{n} \rightarrow \mathbb{R}$, which has zero as a regular value. After possibly considering $-\Phi$ instead of $\Phi$, one can use the normal vector field $\nu_{p}=\nabla \Phi(p)$ for $p \in \mathcal{M}$. In this situation, Lemmas 2.1 and 2.2 show that the exit set test function $u$ is given by

$$
u(p)=\langle\nabla \Phi(p), f(p)\rangle,
$$

and the tangency test function $v$ is given by

$$
v(p)=\langle\nabla \Phi(p), D f(p) f(p)\rangle+\left\langle\operatorname{Hess} \Phi(p) \pi_{p}^{\top} f(p), \pi_{p}^{\top} f(p)\right\rangle .
$$

Notice also that since we use the function $v$ only for points $p \in N^{0}(u)$, one can simplify the definition of $v$ further. If we consider the smooth function $w: \mathcal{M} \rightarrow \mathbb{R}$ given by

$$
w(p)=\langle\nabla \Phi(p), D f(p) f(p)\rangle+\langle\operatorname{Hess} \Phi(p) f(p), f(p)\rangle,
$$

then $w=v$ on the set $N^{0}(u)$. However, the definition of $w$ no longer requires that the vector $f(p)$ in the second term is projected onto the tangent space $T_{p} \mathcal{M}$. This simplifies some of our later computations significantly.

3. Computational Validation of Isolating Blocks. In this section we demonstrate how the theoretical results of the previous section can be turned into computer-assisted proofs for the existence of isolating blocks for ordinary differential equations. Since our method is based on the randomized adaptive nodal domain validation algorithm developed in [3, 7], Section 3.1 will be used to recall this algorithm and its properties. In Sections 3.2 and 3.3 we then show how the algorithm can be extended so that it applies efficiently in our situation. Finally, in Section 3.4 we discuss further possible extensions to the case of isolating blocks whose boundary is only piecewise smooth, and also discuss how different manifold representations have to be treated in different ways.

3.1. Rigorous Approximation of Nodal Domains. Let $\Omega \subset \mathbb{R}^{2}$ denote a closed rectangular domain whose sides are parallel to the coordinate axes. In addition, let $u: \Omega \rightarrow \mathbb{R}$ denote a smooth function. We assume that the function $u$, together with its first-order derivatives, can be rigorously evaluated using interval arithmetic. This means that there are computable real-valued functions $\underline{u}$ and $\bar{u}$ which are defined on the collection of compact subsets of $\Omega$ such that

$$
u(R) \subset[\underline{u}(R), \bar{u}(R)] \quad \text { for all rectangular } R \subset \Omega,
$$

where again we assume that the sides of $R$ are parallel to the coordinate axes. Thus, for every such rectangular subset $R$ we can determine an interval, namely $[\underline{u}(R), \bar{u}(R)]$, which encloses the actual range $u(R) \subset \mathbb{R}$. For more details on interval arithmetic, we refer the reader to [19]. There are a number of software packages available which implement the upper and lower bound functions $\bar{u}$ and $\underline{u}$. For the purposes of this paper, we use the Matlab package INTLAB [20].

Using this notation, the adaptive randomized validation algorithm of [3] can be described as follows. Let $\mathcal{Q}$ denote an initial collection of rectangles with non-overlapping interiors whose union equals $\Omega$. For example, one could simply choose $\mathcal{Q}=\{\Omega\}$. Moreover, let $\mathcal{V}=\emptyset$. Then for every rectangle $R \in \mathcal{Q}$ the following steps are performed: 

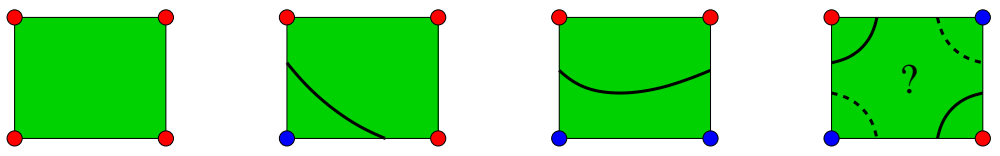

FIG. 1. Sign configurations and the implied nodal line location. From left to right the images correspond to cases (a) through (d) in (V4) of the validation algorithm in [3]. The first three images show all possible corner sign configurations of a rectangle in the final adaptive grid produced by the algorithm, up to rotation, symmetry and sign inversion. The sign configuration shown in the right-most image will never validate, such a rectangle will automatically be subdivided.

(V1) Remove the rectangle $R$ from $\mathcal{Q}$.

(V2) Corner validation: For each of the four corners of $R$, rigorously determine the sign of the function value of $u$ using interval arithmetic, i.e., compute an interval enclosure for the value of $u$ at the corner and test whether it contains zero. If it does for any of the corners, the validation algorithm fails.

(V3) Edge validation: Based on the rigorously verified signs of the function values of $u$ at the corners of $R$, use interval arithmetic to perform one of the following validation tests for each of the four edges of $R$ :

(a) If $u$ has the same sign at the two corners of an edge $E$, check whether zero is contained in the enclosure $[\underline{u}(E), \bar{u}(E)]$.

(b) If $u$ has different signs at the two corners of a horizontal edge $E$, check whether zero is contained in the enclosure $\left[u_{x}(E), \overline{u_{x}}(E)\right]$.

(c) If $u$ has different signs at the two corners of a vertical edge $E$, check whether zero is contained in the enclosure $\left[u_{y}(E), \overline{u_{y}}(E)\right]$.

If zero is contained in the respective range enclosure, then the edge fails the validation step and we procced with step (V5) below. If all four edges pass the validation step, proceed to (V4). Note that if $R$ passes the edge validation step, then every edge contains at most one zero of $u$.

(V4) Face validation: Based on the rigorously verified signs of the function values of $u$ at the corners of $R$, determine which of the four cases shown in Figure 1 occurs - up to sign negation, reflection, and rotation. Then perform the corresponding validation test from the following four possibilities:

(a) If all four signs are the same, determine the enclosure $[\underline{u}(R), \bar{u}(R)]$ for the range $u(R) \subset \mathbb{R}$. If it contains zero, then $R$ fails the validation test, otherwise it passes.

(b) If all but one of the signs are the same, use interval arithmetic as above to show that either $u_{x}(R)$ or $u_{y}(R)$ does not contain zero. If this is the case, $R$ passes the test, otherwise it fails.

(c) If the signs are as in the third image in Figure 1, use interval arithmetic to verify that $u_{y}(R)$ does not contain zero. If this is the case, $R$ passes the test, otherwise it fails. Similarly treat the case when the corners of the left vertical edge have the same sign and the ones on the right edge have the opposite sign by verifying that $u_{x}(R)$ does not contain zero.

(d) In this last case, i.e., the right-most image of Figure 1, $R$ automatically fails the validation test.

We would like to point out that if $R$ passes also the face validation test, then the nodal domain geometry of $u$ in $R$ is qualitatively as shown in the respective image of Figure 1. 
(V5) If one of the validation tests in (V3) or (V4) fails, subdivide $R$ randomly into two subrectangles (more on this below), and add each of these rectangles to $\mathcal{Q}$. If $R$ passes the test, add $R$ to $\mathcal{V}$.

This iteration ends if either $\mathcal{Q}=\emptyset$ is reached, or if one rectangle in $\mathcal{Q}$ is smaller than a pre-specified minimal size, or if (V2) fails. While in the latter two cases the algorithm fails, in the case $\mathcal{Q}=\emptyset$ the algorithm produces a subdivision of $\Omega$ which allows one to qualitatively determine the location of the nodal lines within each rectangle in the final grid. Note that the above version of the validation algorithm is a slight variation of the one described in [3]. As presented here, it can be extended to the higher-dimensional case as well, see [9].

While the details of the random aspects of the algorithm have been explained in [3], we briefly recall what is necessary for our current application. In step (V5) of the algorithm the rectangle $R$ has to be subdivided. This is accomplished by dividing the longer sides of the rectangle into two parts, thereby replacing $R$ by two smaller rectangles. While at first glance it seems prudent to simply divide the longer edge of $R$ into two equal parts, it has been demonstrated in $[3,7]$ that this can lead to validation failures due to grid alignment issues. We therefore subdivide the edge according to ratios which are chosen randomly from a subdivision ratio set. For the purposes of the present work, this set contains the two numbers $(\sqrt{5}-1) / 2$ and $(3-\sqrt{5}) / 2$, i.e., we use the golden ratio for the subdivisions. It was shown in [3] that if the initial set $\Omega$ is a square, then this choice leads to randomized grids with rectangles of only three possible aspect ratios — while still effectively eliminating grid alignment issues.

Notice that in the above description of the algorithm, the validation steps always require to test whether zero is contained in a suitable range enclosure. In fact, upon closer inspection one realizes that it suffices to implement a rigorous interval test of positivity of a function over a rectangle. In [3] this was accomplished in an effective way by extending a branch-and-bound type method due to Skelboe [19]. Finally, it is clear from the form of the algorithm that upon successful completion, one can easily construct a complete representation of the nodal domains of $u$ which is amenable to a subsequent homology computation. This can be done efficiently using the coreduction technique developed in [8].

We close this section with a brief comment on the general applicability of the nodal domain validation algorithm. Even though this was not specifically pointed out in [3], the successful completion of the algorithm establishes the regularity of the value zero, as the following simple lemma shows.

Lemma 3.1. Let $\Omega \subset \mathbb{R}^{2}$ denote a closed rectangular domain and let $u: \Omega \rightarrow \mathbb{R}$ be a twice continuously differentiable function. Furthermore, assume that the algorithm of [3] successfully validates the nodal domains of $u$. Then zero is a regular value of $u$.

Proof. Assume to the contrary that the algorithm validates the nodal domains, but zero is not a regular value of $u$. Then there has to exist a point $x \in \Omega$ such that both $u(x)=0$ and $\nabla u(x)=0$. Let $R \subset \Omega$ denote a closed rectangle in the final grid produced by the algorithm which contains $x$. Due to $u(x)=0$, this rectangle must have been validated as one which contains part of the zero set of $u$, i.e., it must have passed validation steps (V4)(b) or (V4)(c). But then one of the partial derivatives of $u$ must have been either strictly positive or strictly negative on all of $R$. This contradicts $\nabla u(x)=0$.

Thus, in principle the algorithm can only be expected to work if the function $u$ is at least twice continuously differentiable, and if zero is a regular value for $u$, i.e., at every zero of the function $u$ the gradient $\nabla u$ is nonzero. Notice that this also ensures 
that the zero set of $u$ in $\Omega$ consists of nonintersecting $C^{1}$-curves, and it guarantees that small perturbations of $u$ lead to small perturbations of the nodal domains which do not change their topology. This robustness under perturbations is needed. For example, if the zero set contains two intersecting curves, infinitesimally small perturbations could break the crossing in two fundamentally different ways. Notice that this necessarily implies that zero is not a regular value for $u$. Thus, in a generic sense the algorithm can be expected to apply "almost always," and the numerical experiments in [3] attest to that.

3.2. Validation of Exit Sets and the Location of Internal Tangencies. In the form presented in the previous section, the nodal domain validation algorithm is immediately applicable to study two-dimensional manifolds $\mathcal{M}$ in $\mathbb{R}^{3}$, i.e., we now consider the case $n=3$ in Theorem 2.3. For demonstration purposes, in this section we will discuss how the nodal domains of the functions $u$ and $v$ in this theorem, as well as of the simplified test function $w$ from (2.10), can be rigorously validated. For this, consider the vector field $f: \mathbb{R}^{3} \rightarrow \mathbb{R}^{3}$ given by

$$
f(x, y, z)=\left(-\frac{x}{10}+y-x^{3},-x-\frac{y}{10}, 5 z\right),
$$

and let $\mathcal{B} \subset \mathbb{R}^{3}$ denote the region enclosed by an ellipsoid $\mathcal{M}$ centered at the origin, which has major axes of lengths 3,2 , and 2, pointing in the directions of the unit vectors

$$
\left(\begin{array}{c}
1 / \sqrt{5} \\
2 / \sqrt{5} \\
0
\end{array}\right), \quad\left(\begin{array}{c}
-2 / \sqrt{5} \\
1 / \sqrt{5} \\
0
\end{array}\right), \text { and }\left(\begin{array}{l}
0 \\
0 \\
1
\end{array}\right), \quad \text { respectively. }
$$

Elementary algebra shows that the manifold $\mathcal{M}$ is given as the zero set of the smooth function

$$
\Phi(x, y, z)=\frac{2 x^{2}}{9}-\frac{x y}{9}+\frac{5 y^{2}}{36}+\frac{z^{2}}{4}-1,
$$

and therefore the exit set test function $u$ from (2.8) is given by

$$
u(x, y, z)=\frac{1}{180}\left(12 x^{2}-80 x^{4}+34 x y+20 x^{3} y-25 y^{2}+450 z^{2}\right),
$$

for points $(x, y, z) \in \mathcal{M}$. Before we can apply the nodal domain validation algorithm, we need to introduce a two-dimensional parametrization of the manifold. Based on spherical coordinates, we use

$$
\left(\begin{array}{l}
x \\
y \\
z
\end{array}\right)=3 \sin \theta \cos \phi \cdot\left(\begin{array}{c}
1 / \sqrt{5} \\
2 / \sqrt{5} \\
0
\end{array}\right)+2 \sin \theta \sin \phi \cdot\left(\begin{array}{c}
-2 / \sqrt{5} \\
1 / \sqrt{5} \\
0
\end{array}\right)+2 \cos \theta \cdot\left(\begin{array}{l}
0 \\
0 \\
1
\end{array}\right),
$$

with $(\phi, \theta) \in[0,2 \pi] \times[0, \pi]$. Once this formula is plugged into the formula for $u$, one obtains a smooth function defined on the rectangle $\Omega=[0,2 \pi] \times[0, \pi] \subset \mathbb{R}^{2}$, to which the randomized nodal domain validation algorithm from the previous section can be applied.

The result of this validation computation is shown in Figure 2. In both images, the horizontal axis corresponds to $0 \leq \phi \leq 2 \pi$, while the vertical axis is for $0 \leq \theta \leq \pi$. The exit set of the flow generated by (1.1) and (3.1) is shown in dark blue in the left 

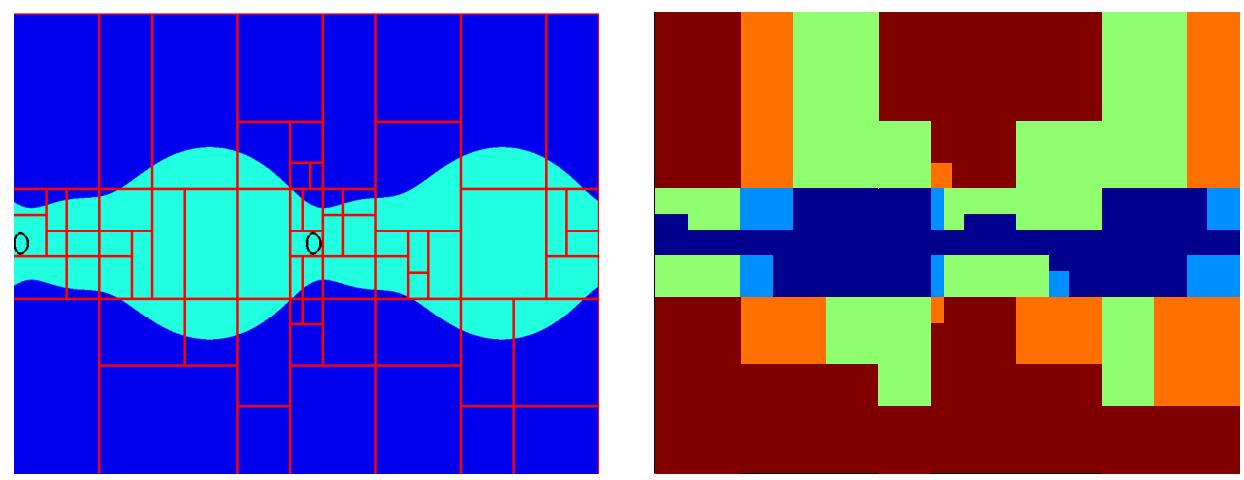

FIG. 2. Isolating block validation for (3.1). The left image shows the exit set of the ellipsoidal isolating block $\mathcal{B}$ described in the text in dark blue, together with the adaptive grid which validates the nodal domains. The black closed loops in the image are the zeros of the tangency test function $w$. The image on the right shows the type of each rectangle in the final grid, color coded as described in the text. In both images, the horizontal axis corresponds to the parametrization variable $0 \leq \phi \leq 2 \pi$, while the vertical axis is for $0 \leq \theta \leq \pi$.
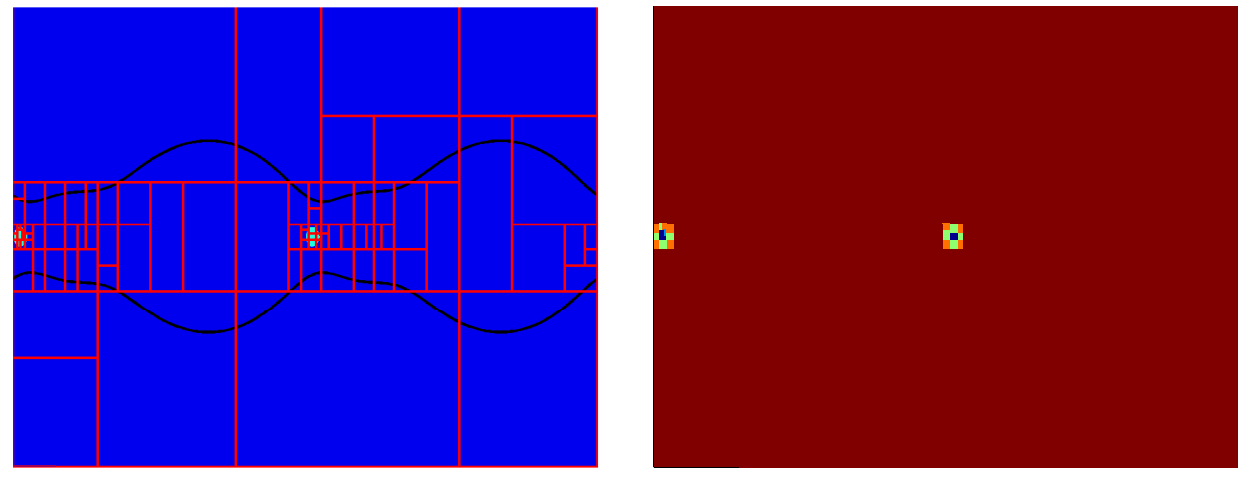

FIG. 3. Isolating block validation for (3.1). The left image shows the positive nodal domain of the tangency test function $w$ of the ellipsoidal isolating block $\mathcal{B}$ described in the text in dark blue, together with the adaptive grid which validates the nodal domains. The black curves in the image are the zeros of the exit set function $u$ as in Figure 2. The image on the right shows the type of each rectangle in the final grid, color coded as described in the text. In both images, the horizontal axis corresponds to the parametrization variable $0 \leq \phi \leq 2 \pi$, while the vertical axis is for $0 \leq \theta \leq \pi$.

image, together with the final adaptive grid produced by the validation algorithm. For each of the rectangles in the final grid, the right image indicates the validation type in the following sense. If the rectangle is red, then $u$ has positive function values at all four corners, if it is orange, exactly three values are positive; green rectangles correspond to two positive and two negative function values as in (V4)(c); finally, light blue indicates one positive and three negative function values, while dark blue implies that $u$ is negative at all four corners of the rectangle. One can clearly see that due to the Skelboe positivity test from [3], the rectangles in the final grid are usually fairly large, and the algorithm does not seem to have to refine excessively.

We now turn our attention to the tangency test function $w$ defined in (2.10). Also this function can be determined explicitly as $u$ before, but we refrain from reproducing the precise formula here, since it is rather long and does certainly not 

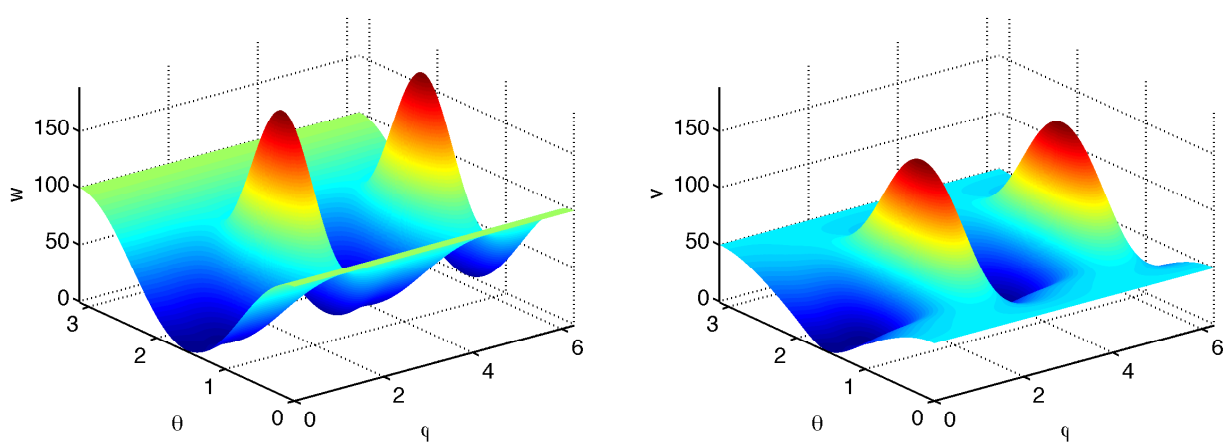

FIG. 4. Isolating block validation for (3.1). The left image shows a graph of the tangency test function $w$ defined in (2.10), while the right image shows the tangency test function $v$ defined in (2.9). The two functions are different, but either can be used to exclude internal flow tangencies at the boundary of the isolating block block $\mathcal{B}$. However, from a computational point of view the function $w$ is easier to work with.

add any additional insight. Suffice it to say that this formula, as well as the one for $v$, can easily be generated using computer algebra software. Applying the nodal domain validation algorithm to $w$ furnishes the images shown in Figure 3. In the left image, the positive nodal domain of $w$ is shown in dark blue, the right image again indicates the validation type of every rectangle in the final grid for the function $w$, using the same color convention as before. In addition, we have included the nodal lines of $w$ as black loops in the left image of Figure 2, and the nodal lines of $u$ as black curves in the left image of Figure 3.

Taken together, the two figures seem to establish the set $\mathcal{B}$ as an isolating block, since the nodal line of $u$ is strictly contained in the positive nodal domain of $w$, see again Theorem 2.3. Note, however, that the adaptive grids produced by the validation algorithm for the functions $u$ and $w$ are almost never aligned. Since the algorithm does not provide precise information on the location of the nodal set within each rectangle, except for which edges it intersects and that it is a simple curve in the interior, overlapping rectangles in the two grids could lead to a situation where it cannot be guaranteed that no internal tangencies occur. In order to address this issue, the validation algorithm needs to be adjusted - and this is the topic of the next section.

To close this section, we briefly return to the tangency test function. In the discussion following Theorem 2.3 we presented two different versions of this function. On the one hand, there is $v$ given in $(2.9)$, which is exactly the function from the theorem, but rewritten for the case of $\mathcal{M}$ being a level set of a smooth function $\Phi$. In this level set framework, we could alter the function in order to avoid projecting the vector $f(p)$ onto the tangent space $T_{p} \mathcal{M}$, giving rise to $w$ in (2.10). In general, the functions $v$ and $w$ are different, see for example Figure 4 for the situation described in the current section. While either function can be used to exclude internal tangencies, the function $w$ does lead to faster computations, sometimes even significantly faster, since the resulting formulas will be less complex. In our situation, the positive nodal domains of $v$ and $w$ are just small perturbations of each other, but both contain the nodal lines of $u$. Note, however, that the definition of $v$ in Theorem 2.3 is given in a general manifold setting, regardless how this manifold is represented. And in this setting the second fundamental form is only defined on the tangent bundle. In addition, as we will see in Section 4, in some cases a level set description of $\mathcal{M}$ is not 
readily available, and in these cases the function $v$ needs to be determined using local coordinate charts. This will be described in more detail in Section 3.4.

3.3. Rigorous Validation of Isolating Blocks. It was pointed out in the last section that in order to establish a set $\mathcal{B}$ as an isolating block one cannot generally just validate the nodal domains of the test functions $u$ and $v$ from Theorem 2.3 separately. Since the resulting adaptive grids will almost certainly not be aligned, overlapping grid rectangles which each contain parts of the zero sets of $u$ and $v$, respectively, would make a definite rigorous answer impossible. Fortunately, this problem can easily be solved by a slight adaption of the nodal domain verification algorithm, which will be presented in the following.

Assume that $\Omega \subset \mathbb{R}^{2}$ is a rectangular coordinate chart over which the test functions $u$ and $v$ are parametrized, i.e., we are given two smooth functions $u, v: \Omega \rightarrow \mathbb{R}$. Then the following algorithm can be used to rigorously establish the assumptions of Theorem 2.3 on $\Omega$. The algorithm makes use of three lists $\mathcal{Q}, \mathcal{V}$, and $\mathcal{T}$ of rectangles, which initially are all empty. Then we proceed through the following steps:

(IB1) Nodal domain validation for $u$ : Apply the nodal domain validation algorithm (V1) through (V5) to the smooth function $u$. If this algorithm fails, then the isolating block validation fails as well. If it succeeds, initialize the list $\mathcal{Q}$ with the generated validated rectangles.

(IB2) Tangency test sweep: For each of the rectangles $R$ in $\mathcal{Q}$, remove the rectangle from $\mathcal{Q}$, and then perform one of the following two steps based on the signs of $u$ at the corners of $R$ :

(a) If the function $u$ has the same sign at all four corners of the rectangle $R$, then add $R$ to the list $\mathcal{V}$.

(b) If the function $u$ takes on both positive and negative function values at the corners of $R$, use interval arithmetic to find a range enclosure $[\underline{v}(R), \bar{v}(R)]$ of $v(R)$. If we have $\underline{v}(R)>0$, then add $R$ to the list $\mathcal{V}$, otherwise, add the rectangle to $\mathcal{T}$.

If the sweep ends with $\mathcal{T}=\emptyset$, then we have shown that $\mathcal{B}$ is an isolating block. Otherwise, we proceed to the next step.

(IB3) Refinement of the nodal domain grid for $u$ : For each of the rectangles in $\mathcal{T}$, remove the rectangle from $\mathcal{T}$, and then apply the nodal domain validation algorithm (V1) through (V5) to the smooth function $\left.u\right|_{R}$ after forcing at least one further subdivision of $R$. If this algorithm fails, then the isolating block validation fails as well. If it succeeds, add the resulting rectangles to the list $\mathcal{Q}$. Once the list $\mathcal{T}$ is empty, return to (IB2) for another sweep of the rectangles in $\mathcal{Q}$.

This iteration ends if either $\mathcal{T}=\emptyset$ is reached, or if a pre-specified maximal number of sweeps (IB2) has been exceeded. While in the latter case the algorithm fails, in the former case the algorithm produces a subdivision of $\Omega$ which allows one to easily verify the assumptions of Theorem 2.3 .

THEOREM 3.2 (Isolating Block Verification). Let $\Omega \subset \mathbb{R}^{2}$ be a rectangular coordinate chart for the manifold $\mathcal{M}=\partial \mathcal{B}$ from Theorem 2.3, and let $u, v: \Omega \rightarrow \mathbb{R}$ denote the smooth exit set and tangency test functions from the theorem, expressed in local coordinates. If the isolating block validation algorithm (IB1) through (IB3) successfully finishes when applied to $u, v$, then (2.6) is satisfied on the closed subset of $\mathcal{M}$ which is parametrized through $\Omega$. Moreover, if the above holds for a finite number of local coordinate charts whose images cover the manifold $\mathcal{M}$, then the set $\mathcal{B}$ is an isolating block for (1.1). 

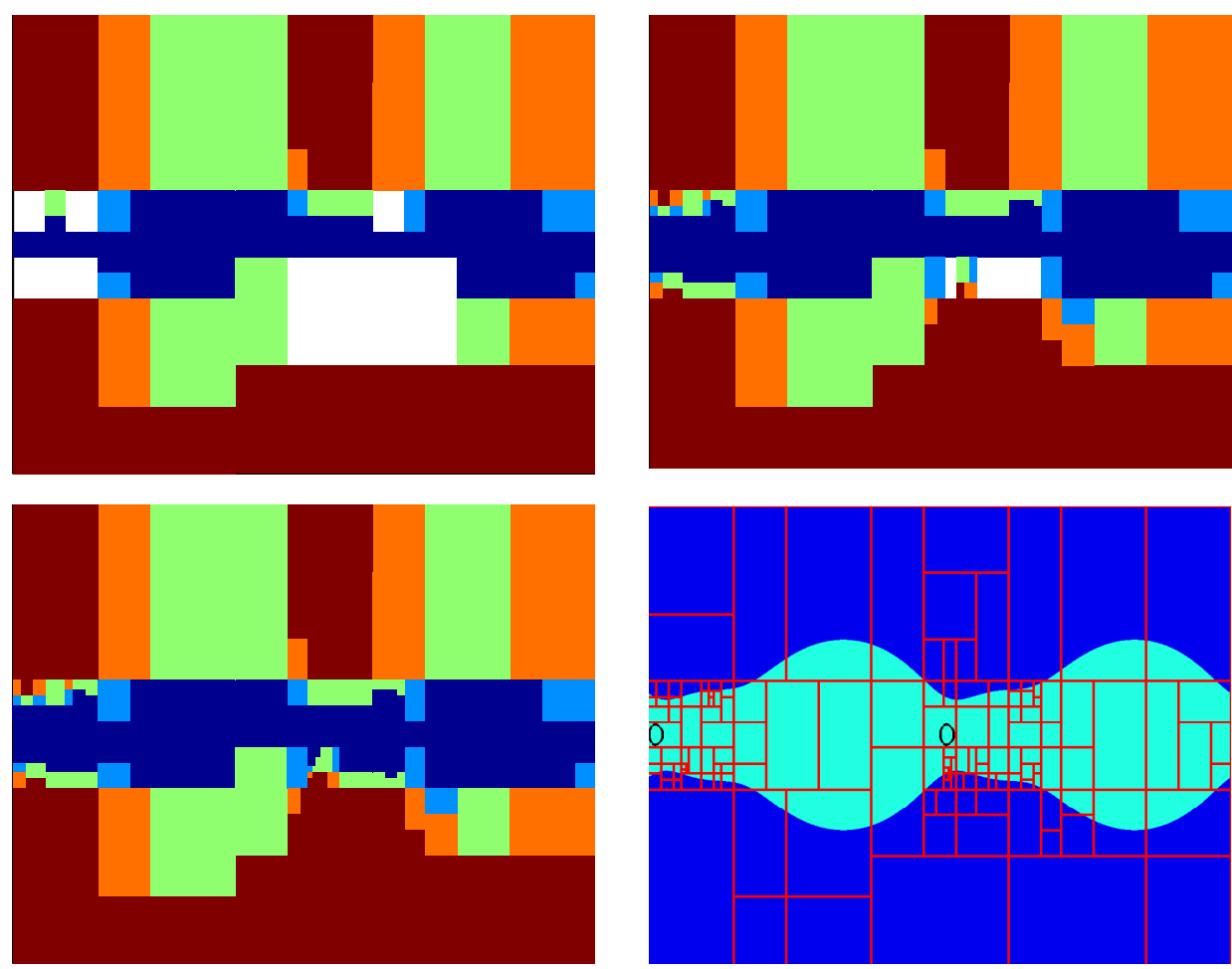

FIG. 5. Isolating block validation for (3.1). From top left to bottom right, the first three images show the state of the isolating block validation algorithm at the end of each step (IB2). In each image, colored rectangles are contained in $\mathcal{V}$, color coded as described in the text; white rectangles are contained in $\mathcal{T}$. The image in the lower right shows the final grid produced by the algorithm, together with the exit set in dark blue, and the zero set of the tangency test function in black. In all four images, the horizontal axis corresponds to the parametrization variable $0 \leq \phi \leq 2 \pi$, while the vertical axis is for $0 \leq \theta \leq \pi$.

Proof. Let $\mathcal{V}$ denote the collection of validated rectangles at the end of the algorithm. Since each of these rectangles has passed the validation step (V4), Lemma 3.1 implies that zero is a regular value over each rectangle, hence over $\Omega$.

Now let $p \in \Omega$ be any point with $u(p)=0$. Then there exists a rectangle $R \in \mathcal{V}$ with $p \in R$. Since $R$ has passed step (IB2) and contains a zero of $u$, it must have passed step (IB2)(b). This shows that $v>0$ on $R$, and therefore we have $v(p)>0$. This completes the proof of the theorem.

We would like to point out that the above algorithm only validates the location of the nodal set of $u$. For the tangency test function $v$, the precise location of its zero set is irrelevant, as long as $v$ can be shown to be strictly positive at zeros of $u$. In fact, the tangency test function $v$ is only evaluated over rectangles $R$, which certifiably contain zeros of $u$. In practice, we perform the positivity test $v(R)>0$ using the Skelboe-type method introduced in [3]. Note, however, that the precise location of the zero set of $u$ is indeed required for the computation of the Conley index, since it determines the exit set $\mathcal{B}^{-}$.

We close this section by applying the isolating block validation algorithm to the example from the previous section. The algorithm validated the set $\mathcal{B}$ as an isolating 
block in three sweeps, leading to the results in Figure 5. From top left to bottom right, the first three images show the state of the isolating block validation algorithm at the end of each sweep (IB2). In each image, colored rectangles are contained in $\mathcal{V}$, and their color indicates at how many corners of the rectangle the function $u$ is positive, in the order red, orange, green, light blue, dark blue from four down to zero. The remaining white rectangles are contained in $\mathcal{T}$. The image in the lower right shows the final grid produced by the algorithm, together with the exit set in dark blue, and the zero set of the tangency test function $w$, which was used instead of $v$, in black.

Notice that these images demonstrate the automated and adaptive nature of the algorithm. After having determined an initial rectangular approximation of the nodal sets of $u$, it then systematically tests for positivity of the tangency test function on all rectangles which contain part of the zero set of $u$. In some cases, these rectangles might contain points at which $v$ is indeed negative, while in others it might just not be possible to establish the positivity of $v$, since the rectangle is still too large. Only when necessary, it then refines the nodal domain approximation locally, and retries the positivity verification of $v$. In this way, the adaptive grid is only refined whenever it is necessary to separate the zero sets of $u$ and $v$.

3.4. Local Coordinate Charts and Piecewise Smooth Boundaries. In Section 3.2 we demonstrated with a specific example how the functions $u$ and $v$, or the modified tangency test function $w$, can be determined if the manifold $\mathcal{M}$ is given as level set of a smooth function $\Phi$. Yet, in many situations such a level set representation might not be available, or might involve a complicated formula. In such cases, one has to revert back to a local coordinate chart and express everything in terms of this coordinate chart. Since the results of Section 2 were formulated in a coordinate-free way, we now briefly summarize how a local parametric description of $\mathcal{M}$ can be used to determine the test functions $u$ and $v$ from Theorem 2.3.

To fix notation, suppose that $\mathcal{M}=\partial \mathcal{B}$ is a smooth manifold which satisfies (A1) through (A3) from Section 2. Suppose further that the mapping $r: \Omega \rightarrow \mathcal{M} \subset \mathbb{R}^{n}$ is a smooth local regular coordinate chart for $\mathcal{M}$, which is defined on a rectangular set $\Omega \subset \mathbb{R}^{n-1}$, i.e., the set $\Omega$ is the product of $n-1$ nontrivial closed intervals. Standard results from differential geometry then imply that the partial derivatives

$$
r_{\theta_{1}}(\theta) \in \mathbb{R}^{n}, \ldots, r_{\theta_{n-1}}(\theta) \in \mathbb{R}^{n} \quad \text { with } \quad \theta=\left(\theta_{1}, \ldots, \theta_{n-1}\right) \in \Omega
$$

form a usually not normalized basis for the tangent space $T_{p} \mathcal{M}$ at $p=r(\theta)$. In the special case $n=3$, one can therefore explicitly compute a normal vector $\nu_{p}$ as in (A2) by letting

$$
\nu_{p}= \pm\left(r_{\theta_{1}}(\theta) \times r_{\theta_{2}}(\theta)\right), \quad \text { or normalized as } \quad \nu_{p}= \pm \frac{r_{\theta_{1}}(\theta) \times r_{\theta_{2}}(\theta)}{\left\|r_{\theta_{1}}(\theta) \times r_{\theta_{2}}(\theta)\right\|},
$$

where the sign is chosen in such a way that $\nu_{p}$ points away from $\mathcal{B}$. In the general case, the normal vector could be found by solving an appropriate linear system. With $\nu_{p}$ at hand, the test function $u$ from (2.4) can then be written in local coordinates as

$$
u\left(\theta_{1}, \ldots, \theta_{n-1}\right)=\left\langle f\left(r\left(\theta_{1}, \ldots, \theta_{n-1}\right)\right), \nu_{r\left(\theta_{1}, \ldots, \theta_{n-1}\right)}\right\rangle,
$$

for $\left(\theta_{1}, \ldots, \theta_{n-1}\right) \in \Omega$.

We now turn our attention to the tangency test function $v$, in particular, to the local representation of the second fundamental form. Let $\theta=\left(\theta_{1}, \ldots, \theta_{n-1}\right) \in \Omega$ be 
fixed, and define $p=r\left(\theta_{1}, \ldots, \theta_{n-1}\right) \in \mathcal{M}$. By solving the appropriate linear system, one can find real numbers $\alpha_{1}(p), \ldots, \alpha_{n-1}(p) \in \mathbb{R}$ and $\beta(p) \in \mathbb{R}$ such that

$$
f(p)=\beta(p) \nu_{p}+\sum_{k=1}^{n-1} \alpha_{k}(p) r_{\theta_{k}}(\theta) .
$$

Then the projection $\pi_{p}^{\top} f(p)$ is given by the sum in the previous expression, and as described in [10, pp. 126-128], the second fundamental form of $\mathcal{M}$ evaluated at this projection takes the form

$$
\mathbb{I}\left(\pi_{p}^{\top} f(p), \pi_{p}^{\top} f(p)\right)=\sum_{k, \ell=1}^{n-1}\left\langle r_{\theta_{k} \theta_{\ell}}(\theta), \nu_{p}\right\rangle \cdot \alpha_{k}(p) \alpha_{\ell}(p) .
$$

This finally furnishes the tangency test function $v$ from (2.5) in local coordinates in the form

$$
v(\theta)=\left\langle D f(r(\theta)) f(r(\theta)), \nu_{r(\theta)}\right\rangle-\sum_{k, \ell=1}^{n-1}\left\langle r_{\theta_{k} \theta_{\ell}}(\theta), \nu_{r(\theta)}\right\rangle \cdot \alpha_{k}(r(\theta)) \alpha_{\ell}(r(\theta)),
$$

for $\theta=\left(\theta_{1}, \ldots, \theta_{n-1}\right) \in \Omega$. Notice that in this form, the Hessian matrix of the parametrization $r$ appears explicitly in the formula. Moreover, this formula is valid even if the normal vector $\nu$ is not normalized.

To close this section, we briefly indicate a possible extension of our approach. So far, we have always assumed that the boundary of the potential isolating block $\mathcal{B}$ is given by a smooth manifold. For some applications, however, it might be useful to consider regions with piecewise smooth boundary $\mathcal{M}$. Of course, on each of the maximal smooth subsets $\mathcal{M}_{k}$ of $\mathcal{M}$, we can still use the isolating block validation algorithm to verify the assumptions of Theorem 2.3. But what can be said about the points in $\mathcal{M}$ at which the smooth submanifolds with boundary meet? For this, let $\mathcal{M}_{1} \subset \mathcal{M}$ and $\mathcal{M}_{2} \subset \mathcal{M}$ denote two closed smooth submanifolds of $\mathcal{M}$ with boundary, and assume that their intersection is contained in each of their boundaries. Furthermore, assume that both submanifolds satisfy the assumptions of Theorem 2.3, and that along their intersection, the region $\mathcal{B}$ is convex in the following sense, which is due to [11]:

(C) If $p \in \mathcal{M}_{1} \cap \mathcal{M}_{2}$, then there exists a neighborhood $U$ of $p$ in $\mathcal{M}$, such that for any point $p_{1} \in \mathcal{M}_{1} \cap U$ and any point $p_{2} \in \mathcal{M}_{2} \cap U$ their connecting line segment is completely contained in $\mathcal{B}$.

In this case, at all points $p \in \mathcal{M}_{1} \cap \mathcal{M}_{2}$ the flow of (1.1) either enters or exits in a non-trivial way, i.e., we have $p \in \mathcal{B}^{+} \backslash \mathcal{B}^{-}$or $p \in \mathcal{B}^{-} \backslash \mathcal{B}^{+}$, respectively, or there exists a time $T>0$ such that $\varphi([-T, T], p) \cap \mathcal{B}=\{p\}$. In other words, it suffices to study only the smooth submanifolds up to and including their boundaries to establish an isolating block. Formulating a corresponding result in general dimensions appears to be technically involved, even though in special cases it is easy to show that the above statement holds. We therefore refrain from presenting a general result.

4. Three Numerical Case Studies. In this final section of the paper we present a number of examples which illustrate the verification procedure of the last section. It is our hope that these examples demonstrate both the simplicity of our approach, as well as its applicability. In particular, we will demonstrate that even if a candidate set $\mathcal{B}$ does not turn out to be an isolating block, the output of the validation algorithm (IB1) through (IB3) can provide crucial information as to how the set has to be changed in order to furnish an isolating block. 

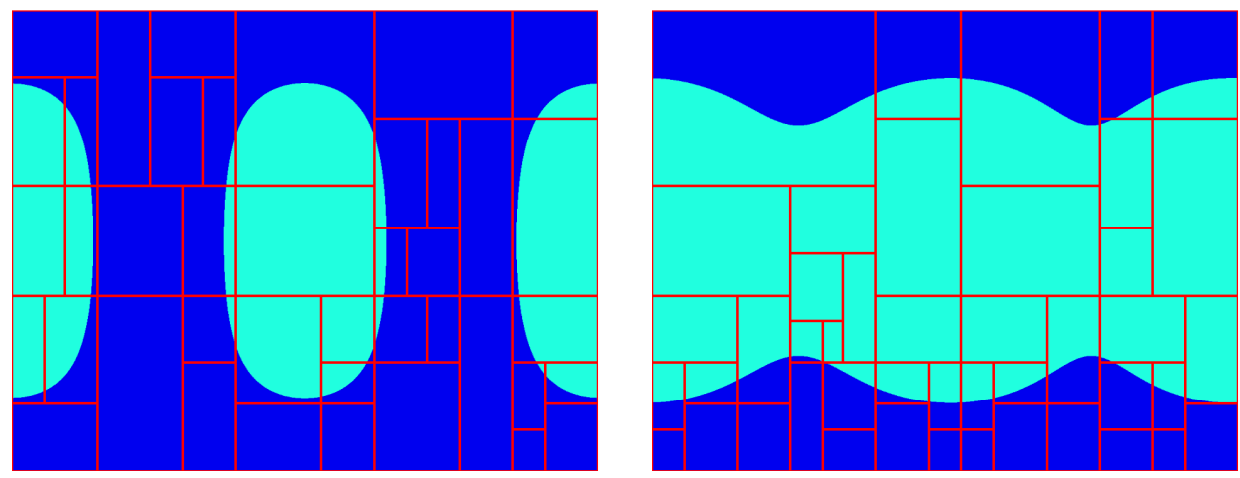

FIG. 6. Isolating block validation for (4.1). The left and right images show the exit sets of the isolating blocks $\mathcal{A}_{0}$ and $\mathcal{A}_{2}$, respectively, in dark blue; the final adaptive grid produced by the isolating block validation algorithm is shown in red. In both cases, the modified tangency test function $w$ is strictly positive on the whole domain. The horizontal axis corresponds to the variable $0 \leq \phi \leq 2 \pi$ in both images, while the vertical axis is for $0 \leq \theta \leq \pi$.

4.1. A Simple Gradient System. Our first example is taken from Eberlein's thesis [11] and is given by the system

$$
\begin{aligned}
& \dot{x}=2 x(z-y), \\
& \dot{y}=1+z-x^{2}, \\
& \dot{z}=-1+y+x^{2} .
\end{aligned}
$$

One can easily see that this system is a gradient system with associated potential

$$
V(x, y, z)=x^{2} y-x^{2} z-y z-y+z,
$$

i.e., the right-hand side of $(4.1)$ is given by $-\nabla V(x, y, z)$. Note that contrary to our presentation, in [11] the potential $-V$ is considered and orbits move to increase $V$. Instead, we have adopted the standard definition of a gradient system where the flow of (4.1) decreases the potential $V$.

One can easily verify that the system (4.1) has exactly three equilibrium solutions given by $p_{0}=(-1,0,0), p_{1}=(1,0,0)$, and $p_{2}=(0,1,-1)$. All of these are hyperbolic with indices 2,2 , and 1 , respectively. Our validation algorithm can be used to obtain these indices if one chooses for example the following three neighborhoods:

- The set $\mathcal{A}_{0}$ is a 2-norm ellipsoid whose principal axes are pointing in the direction of the three eigenvectors of the linearization of (4.1) at $p_{0}$. The length of each principal axis is given by $3 / 20$ divided by the absolute value of the corresponding eigenvalue.

- The set $\mathcal{A}_{1}$ is constructed analogously to $\mathcal{A}_{0}$, but for $p_{1}$ instead of $p_{0}$.

- The set $\mathcal{A}_{2}$ is a sphere of radius $1 / 5$ centered at $p_{2}$.

The boundary of each of these sets is parameterized using spherical coordinates as described in Section 3.2, and the algorithm described in Section 3.3 rigorously verifies them as isolating blocks with the anticipated indices

$$
C H_{*}\left(\mathcal{A}_{0}\right) \simeq C H_{*}\left(\mathcal{A}_{1}\right) \simeq(0,0, \mathbb{Z}, 0, \ldots) \text { and } C H_{*}\left(\mathcal{A}_{2}\right) \simeq(0, \mathbb{Z}, 0,0, \ldots) .
$$

The output of the isolating block validation algorithm for the sets $\mathcal{A}_{0}$ and $\mathcal{A}_{2}$ is shown in the left and right images of Figure 6, respectively. In both cases, the modified 

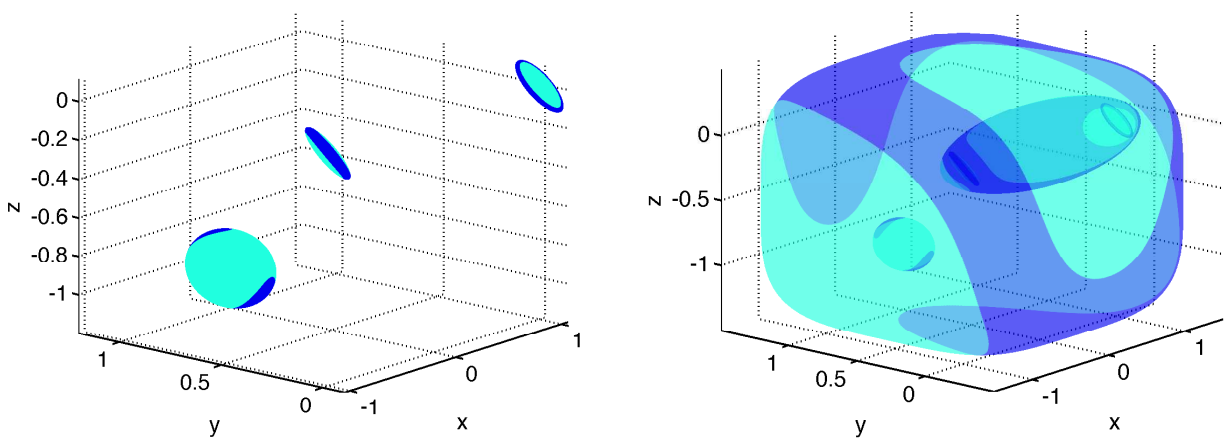

FIG. 7. Visualization of several validated isolating blocks for the flow of (4.1). The depicted surfaces are the boundaries of the isolating blocks, exit sets are shown in dark blue, while the flow enters the blocks in the light blue regions. The left image shows the isolating blocks $\mathcal{A}_{0}, \mathcal{A}_{1}$, and $\mathcal{A}_{2}$ enclosing the equilibria $p_{0}, p_{1}$, and $p_{2}$, respectively. In addition to these, the right image also shows the isolating blocks $\mathcal{A}_{01}$ and $\mathcal{A}_{012}$ described in the text.

tangency test function $w$ defined in (2.10) turns out to be strictly positive over the parameterization domain $\Omega=[0,2 \pi] \times[0, \pi]$. The results for $\mathcal{A}_{1}$ were omitted, since this block validated similar to $\mathcal{A}_{0}$. All three isolating blocks are shown in the left image of Figure 7. We would like to point out that the specific forms of these sets as ellipsoids or sphere are not essential. We could have easily chosen spheres in all three cases. Note, however, that at the equilibria $p_{0}$ and $p_{1}$, the exit set boundary intersects the three lines through each equilibrium which are parallel to the coordinate axes, due to inherent symmetries in (4.1). In order to use the boundary parameterization as in Section 3.2, one therefore has to slightly rotate the coordinate system. Since the "north" and "south pole" of the parameterization will be represented by the line segments $\theta=0$ and $\theta=\pi$, they have to be contained either in the interior of the exit set or of the entry set.

As a gradient system with three hyperbolic fixed points, the only other type of globally bounded orbits are heteroclinic orbits between these equilibrium solutions. One can readily see that there is no possibility of a heteroclinic between the stationary states $p_{0}$ and $p_{1}$, since the plane $x=0$ is invariant under the flow and the two equilibria lie on either side of it. But what about heteroclinics between $p_{0}$ and $p_{2}$, or between $p_{1}$ and $p_{2}$ ? In [11] the existence of at least one of these connections was established by using two polygonal isolating blocks - one which contains $p_{0}$ and $p_{1}$, and one which contains all three equilibria. Similarly, we consider the following two neighborhoods:

- The set $\mathcal{A}_{01}$ is a 2 -norm ellipsoid centered at the origin whose principal axes are pointing in the coordinate directions $x, y$, and $z$ and have lengths $6 / 5$, $3 / 10$, and $3 / 10$, respectively.

- In contrast, the set $\mathcal{A}_{012}$ is a 4-norm ellipsoid centered at the point $(0,1 / 2,-1 / 2)$ whose principal axes are pointing in the coordinate directions $x, y$, and $z$ and have lengths $3 / 2,1$, and 1 , respectively.

Our algorithm confirms that these sets are in fact isolating blocks, and they are shown in the right image of Figure 7 . The boundary of each of these sets is parameterized using spherical coordinates $\phi$ and $\theta$ as described in Section 3.2, and the validated exit sets as functions of $\phi$ and $\theta$ are depicted in Figures 8 and 9 for the isolating blocks $\mathcal{A}_{01}$ and $\mathcal{A}_{012}$, respectively. In both cases, the tangency test function $w$ was strictly positive over the domain. From these images one can readily deduce that the 

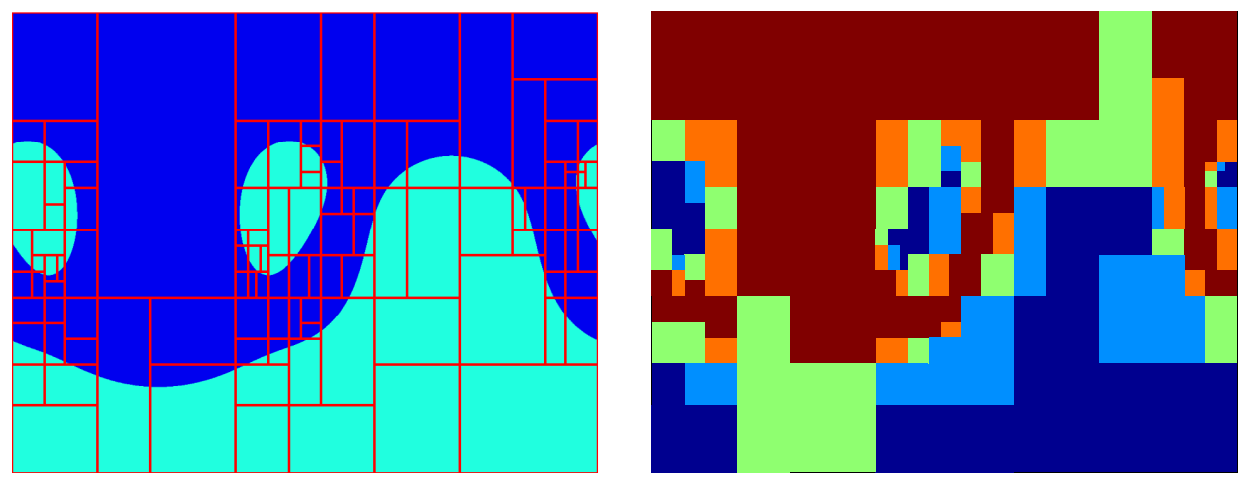

FIG. 8. Isolating block validation for (4.1). The left image shows the exit set of the isolating block $\mathcal{A}_{01}$ in dark blue, as well as the final adaptive grid produced by the validation algorithm. The image on the right shows the type of each rectangle in the final grid, color coded according to the number of positive function values of $u$ at the corners of each rectangle, with red, orange, green, light blue, and dark blue corresponding to four, three, two, one, and zero, respectively. In both images, the horizontal axis is for the variable $0 \leq \phi \leq 2 \pi$, while the vertical axis is for $0 \leq \theta \leq \pi$. These computations imply that the Conley index of $\mathcal{A}_{01}$ is the sum of the Conley indices of $\overline{\mathcal{A}}_{0}$ and $\mathcal{A}_{1}$, i.e., it is given by $\left(0,0, \mathbb{Z}^{2}, 0, \ldots\right)$.
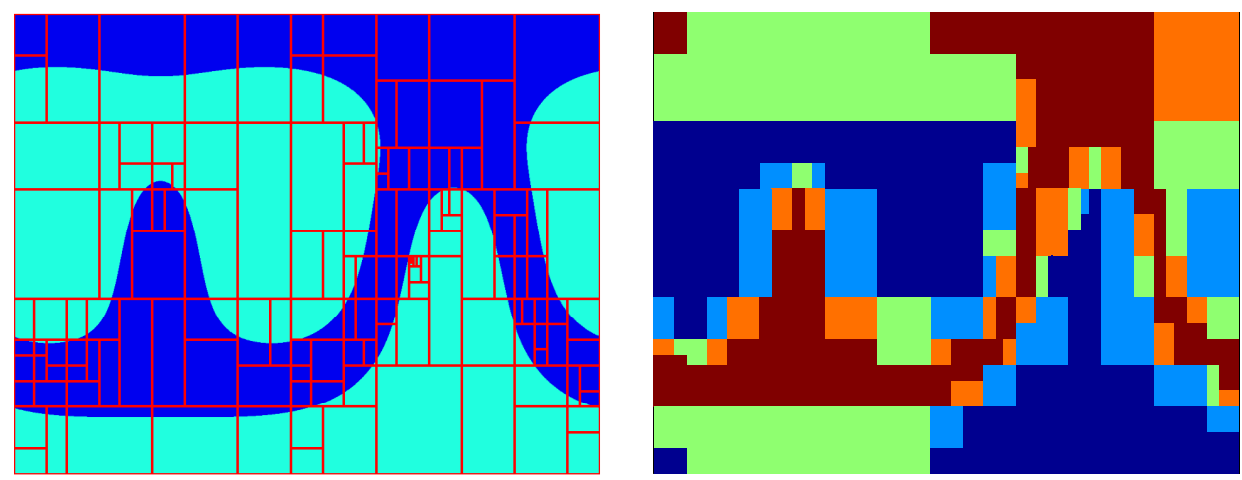

FIG. 9. Isolating block validation for (4.1). The two images contain the output of the isolating block validation algorithm applied to $\mathcal{A}_{012}$, analogous to Figure 8. The associated Conley index is given by $(0,0, \mathbb{Z}, 0, \ldots)$, i.e., the index of an index two equilibrium.

associated Conley indices are given by

$$
C H_{*}\left(\mathcal{A}_{01}\right) \simeq\left(0,0, \mathbb{Z}^{2}, 0, \ldots\right) \text { and } C H_{*}\left(\mathcal{A}_{012}\right) \simeq(0,0, \mathbb{Z}, 0, \ldots) .
$$

This furnishes

$$
C H_{*}\left(\mathcal{A}_{01}\right) \oplus C H_{*}\left(\mathcal{A}_{2}\right) \not ॅ C H_{*}\left(\mathcal{A}_{012}\right),
$$

which as in [11] implies the existence of a heteroclinic connection between the isolated invariant set in $\mathcal{A}_{01}$ — which is comprised of $p_{0}$ and $p_{1}$ - and the equilibrium $p_{2}$.

Yet, we can easily say more than that. If we can find two isolating blocks, one encapsulating only $p_{0}$ and $p_{2}$, and the other only containing $p_{1}$ and $p_{2}$, in such a way that their Conley indices are different from

$$
C H_{*}\left(\mathcal{A}_{k}\right) \oplus C H_{*}\left(\mathcal{A}_{2}\right) \simeq(0, \mathbb{Z}, \mathbb{Z}, 0, \ldots), \quad \text { for } \quad k=0,1,
$$



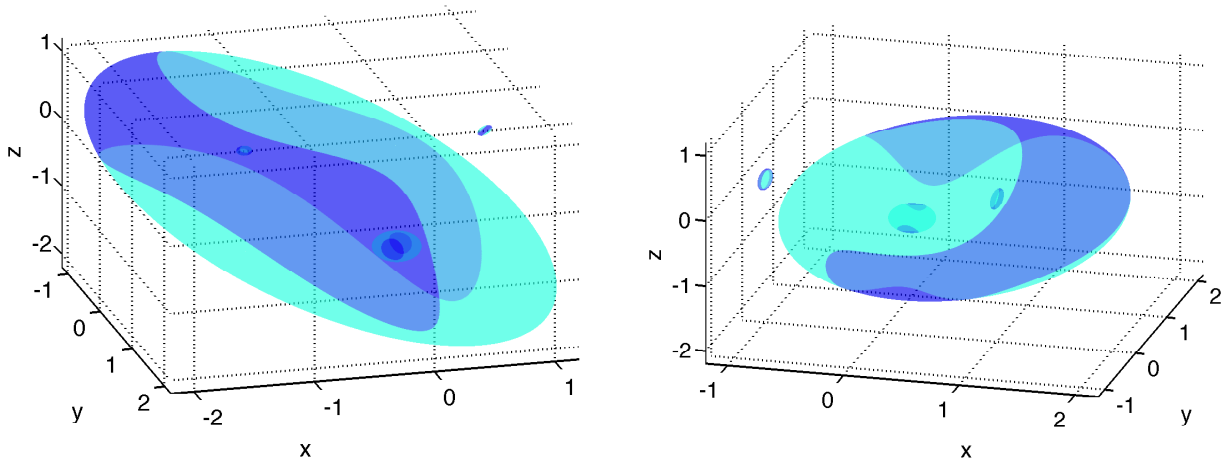

FIG. 10. Visualization of validated isolating blocks $\mathcal{A}_{02}$ (left image) and $\mathcal{A}_{12}$ (right image) for the flow of (4.1). These isolating blocks establish the existence of heteroclinic orbits from $p_{0}$ to $p_{2}$, and from $p_{1}$ to $p_{2}$, respectively. In addition, both images depict the isolating blocks $\mathcal{A}_{0}, \mathcal{A}_{1}$, and $\mathcal{A}_{2}$ enclosing the three equilibria.
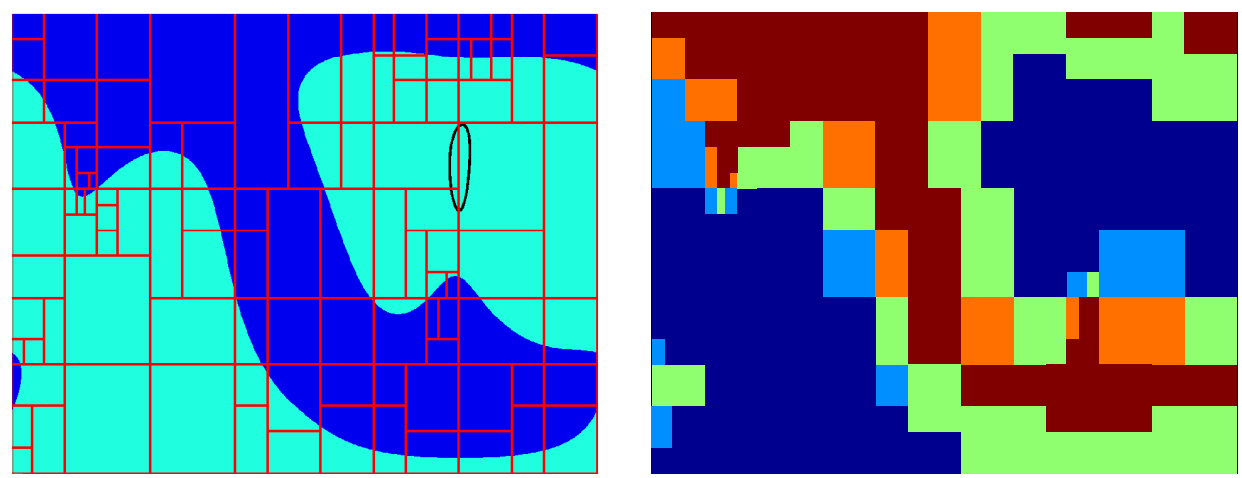

FIG. 11. Isolating block validation for (4.1). The images contain the output of the isolating block validation algorithm applied to $\mathcal{A}_{12}$, as in Figure 8. The associated Conley index is trivial. In the left image, the zero set of the modified tangency test function $w$ is shown in black; outside of this loop, the function $w$ is positive.

then the same reasoning as above proves the existence of heteroclinics between both $p_{0}$ and $p_{2}$, and between $p_{1}$ and $p_{2}$. This can be accomplished using the following sets:

- The sets $\mathcal{A}_{02}$ and $\mathcal{A}_{12}$ are congruent 2-norm ellipsoids which are centered at the points $(-1 / 2,1 / 2,-1 / 2)$ and $(1 / 2,1 / 2,-1 / 2)$, respectively. Their major axes have lengths $12 / 5,6 / 5,6 / 5$ and point in the directions of the vectors

$$
\left(\begin{array}{c} 
\pm 1 / \sqrt{3} \\
1 / \sqrt{3} \\
-1 / \sqrt{3}
\end{array}\right), \quad\left(\begin{array}{c}
\mp 1 / \sqrt{2} \\
1 / \sqrt{2} \\
0
\end{array}\right), \quad \text { and } \quad\left(\begin{array}{c}
\mp 1 / \sqrt{6} \\
-1 / \sqrt{6} \\
-2 / \sqrt{6}
\end{array}\right), \quad \text { respectively. }
$$

Again, our validation algorithm confirms these sets as isolating blocks, and they are shown in the left and right images of Figure 10. The boundary of each of these sets is again parameterized using spherical coordinates $\phi$ and $\theta$, and for $\mathcal{A}_{12}$ the validated exit set is shown in Figure 11. This time, the tangency test function $w$ has a nontrivial negative nodal domain given by the interior of the black loop depicted in the left image of Figure 11 - yet this loop lies in the interior of the entry set. For both isolating 
blocks, the associated Conley index is trivial, and this furnishes the existence of both above-mentioned heteroclinics.

4.2. Asymptotic Behavior of Ground States. In [14], Hulshof \& van der Vorst studied the asymptotic behavior of ground states of coupled semilinear Poisson equations. Their approach could reduce the problem to establishing the existence of a certain heteroclinic orbit in a three-dimensional ordinary differential equation. This equation was later considered by Boczko et al. [2] as a test case for their polygonal flow approximations. In the following, we will show that our isolating block validation algorithm can be applied in this situation as well. We consider the ordinary differential equation

$$
\begin{aligned}
& \dot{x}=-x(x+1)-z, \\
& \dot{y}=y(2+6 x-y)+3 x+z, \\
& \dot{z}=z(2-x+5 y) .
\end{aligned}
$$

One can easily verify that this ordinary differential equation has equilibria at the points $p_{0}=(0,0,0)$ and $p_{1}=(-1,-1,0)$, and our goal is to establish the existence of a heteroclinic orbit from $p_{0}$ to $p_{1}$. Note, however, that these are not the only stationary states of (4.3). Additional rest states can be found at $(2,0,-6),(0,2,0)$, $(-1,-3,0)$, and $(-3,-1,-6)$. None of these will be relevant in the following, as they will lie outside all constructed isolating blocks.

As in the first example, finding isolating blocks around the equilibrium solutions is an easy task. We chose two balls $\mathcal{B}_{k}$ of radius $3 / 20$ centered at $p_{k}+(0,0,11 / 100)$, for $k=0,1$, and our verification method confirms them as isolating blocks. Finding an isolating block $\mathcal{B}_{01}$ encompassing both $p_{0}$ and $p_{1}$, as well as the anticipated heteroclinic orbit proves to be slightly more involved. As shown in [2], the orbit has the general shape of a parabola connecting the equilibria, and reaching almost to a height of 1 . Thus, it seems reasonable to construct $\mathcal{B}_{01}$ as the $\varrho$-neighborhood of the parabolic space curve

$$
r(\theta)=\left(\begin{array}{c}
\theta-1 \\
\theta-1 \\
4 h \theta(1-\theta)+c
\end{array}\right) \quad \text { for } \quad 0 \leq \theta \leq 1
$$

for suitable constants $c$ and $h$, and with a suitable circular cross section of radius $\varrho$. We parameterize the surface of the tube in the following way. For $0 \leq \theta \leq 1$ the standard Frenet-Seret frames are used to describe the circular cross section of $\mathcal{B}_{01}$ with the plane through $r(\theta)$ and spanned by the normal and binormal vectors at this point. In this way, the cylindrical surface is parameterized using $0 \leq \theta \leq 1$ along the curve, and using $0 \leq \phi \leq 2 \pi$ along the circular cross section. For $-1 / 2 \leq \theta<0$ and for $1<\theta \leq 3 / 2$ we use standard spherical coordinates to parameterize the spherical caps at the ends of $\mathcal{B}_{01}$.

As a first attempt, we choose $h=1, c=0$, and thickness $\varrho=1 / 5$. The corresponding exit set and the nodal domains of the tangency test function $v$ are shown in the top row of Figure 12. Note that while the exit set would give $\mathcal{B}_{01}$ a trivial Conley index, thus establishing the connecting orbit, it turns out that for these parameter values $\mathcal{B}_{01}$ is not in fact an isolating block - the nodal line associated with the exit set intersects the yellow region of internal tangencies.

Upon closer inspection one can see that this yellow region is located at the bottom bend of the tubular neighborhood, where the relatively high curvature of $r(\theta)$ is larger 

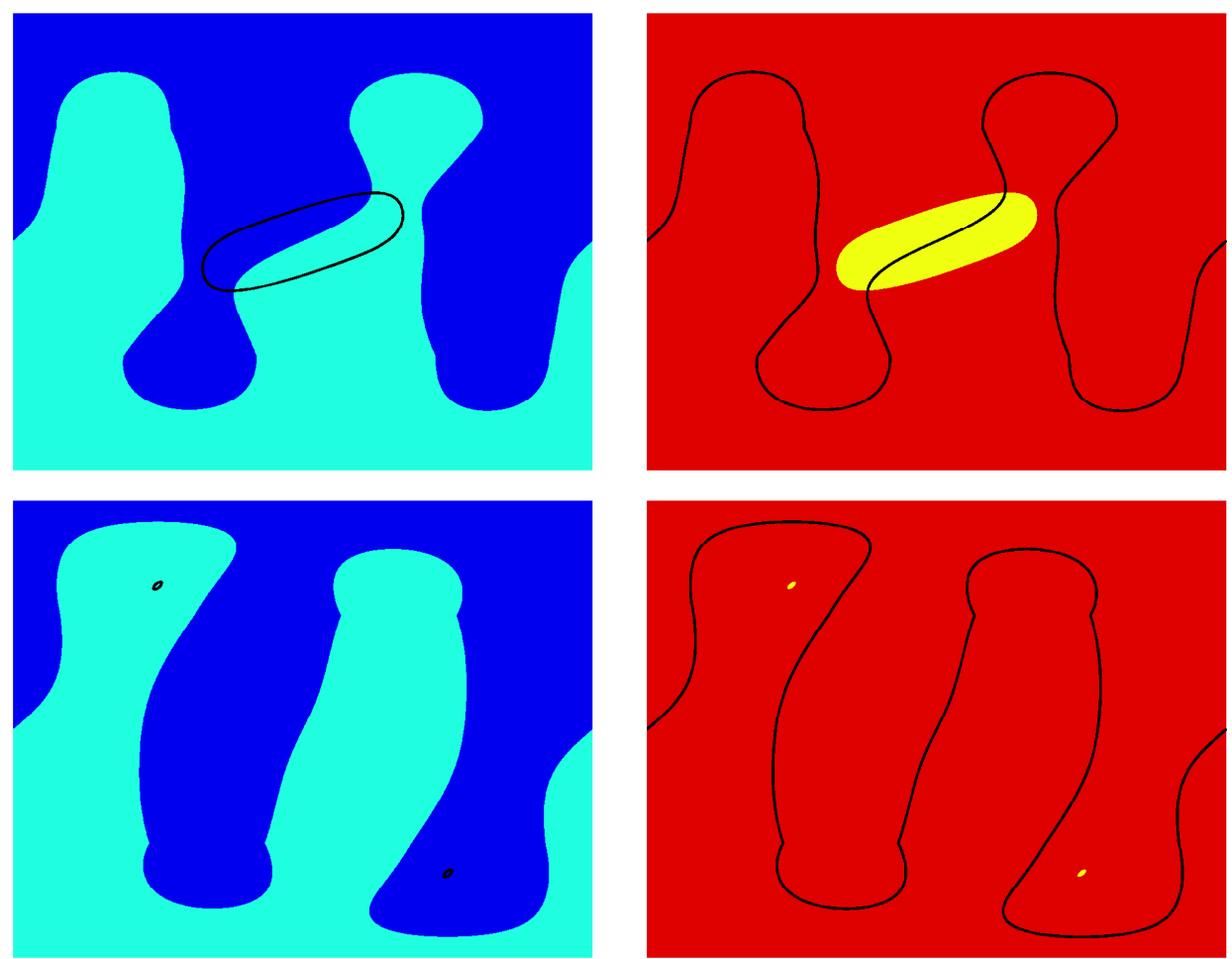

FIG. 12. Two attempts at finding an isolating block for the ground state example (4.3). The top row shows the exit set and the nodal domains of the tangency test function for a tubular neighborhood of the parabolic space curve (4.4) with $h=1$ and $c=0$. Since the boundary of the exit set intersects with the yellow region, there are internal tangencies - and this neighborhood is not an isolating block. The bottom row shows the exit set and the tangency test function for the tubular neighborhood $\mathcal{B}_{01}$ of (4.4) with $h=1 / 2$ and $c=1 / 5$. This time, one obtains an isolating block. In all images, the horizontal axis corresponds to the variable $0 \leq \phi \leq 2 \pi$, while the vertical axis corresponds to $-1 / 2 \leq \theta \leq 3 / 2$, as described in the text.

than the curvature of the flow. Thus, in order to achieve an isolating neighborhood one needs to decrease the curvature of $r(\theta)$ at the bend, while still maintaining isolation of the heteroclinic orbit. This can be accomplished by decreasing $h$, while at the same time increasing $c$ and possibly also $\varrho$. More precisely, if we choose $h=1 / 2, c=1 / 5$, and thickness $\varrho=1 / 4$, we obtain a tubular neighborhood $\mathcal{B}_{01}$ whose exit set and the nodal domains of the tangency test function $v$ are shown in the bottom row of Figure 12. In fact, even though we are not including the actual output, applying the isolating block validation algorithm from Section 3.3 does indeed establish the new set as an isolating block with trivial Conley index which contains $p_{0}$ and $p_{1}$. Since these equilibria have indices 2 and 1 , the maximal invariant set in $\mathcal{B}_{01}$ has to contain at least one additional orbit. In the left image of Figure 13 the three isolating blocks $\mathcal{B}_{0}$, $\mathcal{B}_{1}$, and $\mathcal{B}_{01}$ are shown, and the right image contains a few flow curves of (4.3) which are tangential to $\mathcal{B}_{01}$ (red indicates negative direction of time, black is for positive time).

The only missing piece is to identify the additional part of the isolated invariant set as a connecting orbit. For this, one just has to note that in the quadrant $2-x+5 y>0$ and $z>0$, whose closure contains $p_{0}$, the $z$-component of the flow of (4.3) is strictly 

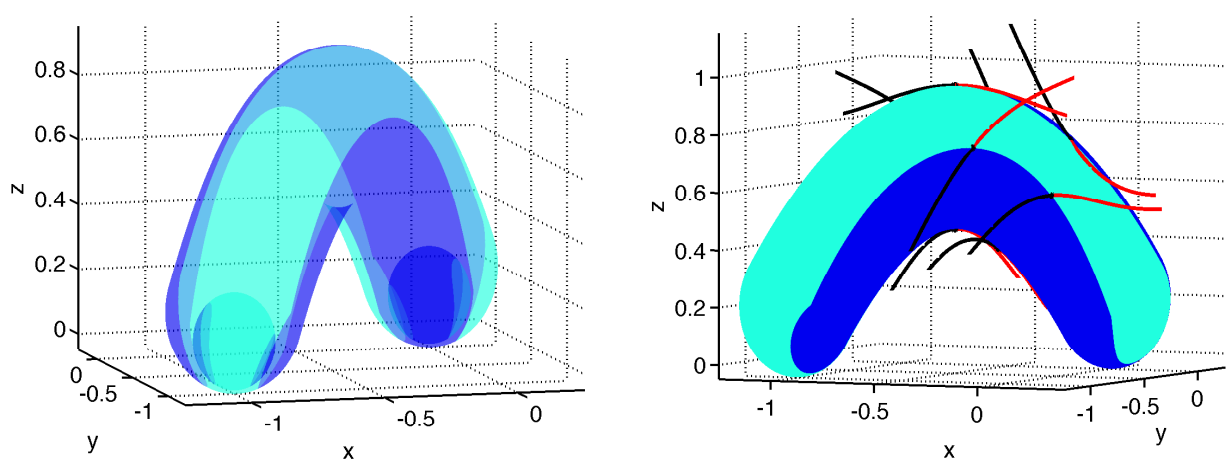

FIG. 13. Visualization of the isolating block $\mathcal{B}_{01}$ which proves the existence of a heteroclinic orbit from $p_{0}$ to $p_{1}$. The left image shows $\mathcal{B}_{01}$, together with two spherical isolating blocks $\mathcal{B}_{0}$ and $\mathcal{B}_{1}$ around $p_{0}$ to $p_{1}$, respectively. The right image shows the isolating block $\mathcal{B}_{01}$ again, but this time with selected solution segments of (4.3) which are outer tangencies to the isolating block.
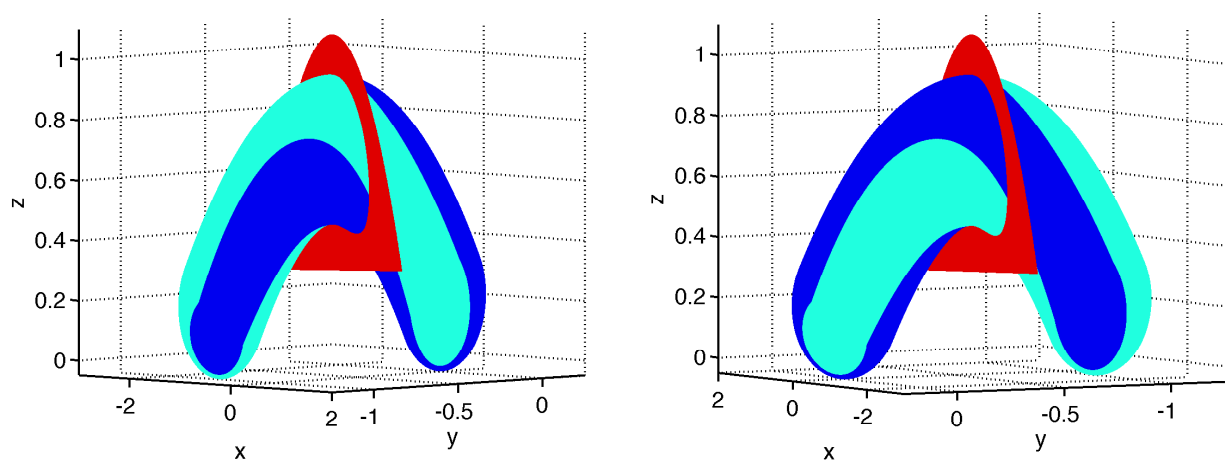

FIG. 14. Visualization of the isolating block $\mathcal{B}_{01}$ for (4.3) from two perspectives. The red region is the part of the plane $2-x+5 y=0$ in which the flow field of (4.3) is transverse to the plane with decreasing $x$ - and $y$-coordinates. As shown in the text, this additional piece of information is necessary to establish the heteroclinic orbit between $p_{0}$ and $p_{1}$.

increasing, while in the quadrant $2-x+5 y<0$ and $z>0$, whose closure contains $p_{1}$, the $z$-component is strictly decreasing. As shown in Figure 14, the part of the plane $2-x+5 y=0$ in which the flow field of (4.3) is transverse to the plane with decreasing $x$ - and $y$-coordinates contains a complete cross section of $\mathcal{B}_{01}$. In other words, the only possible additional full orbit in $\mathcal{B}_{01}$ is a heteroclinic orbit which leaves $p_{0}$ with increasing $z$-component until it traverses the red region of Figure 14, and then drops down towards $p_{1}$.

4.3. A Planar Hopf Bifurcation. Our final example is concerned with an essentially planar system undergoing a Hopf bifurcation. The planar version of this example was considered in the work of Eberlein \& Scheurle [12]. Since our focus is 

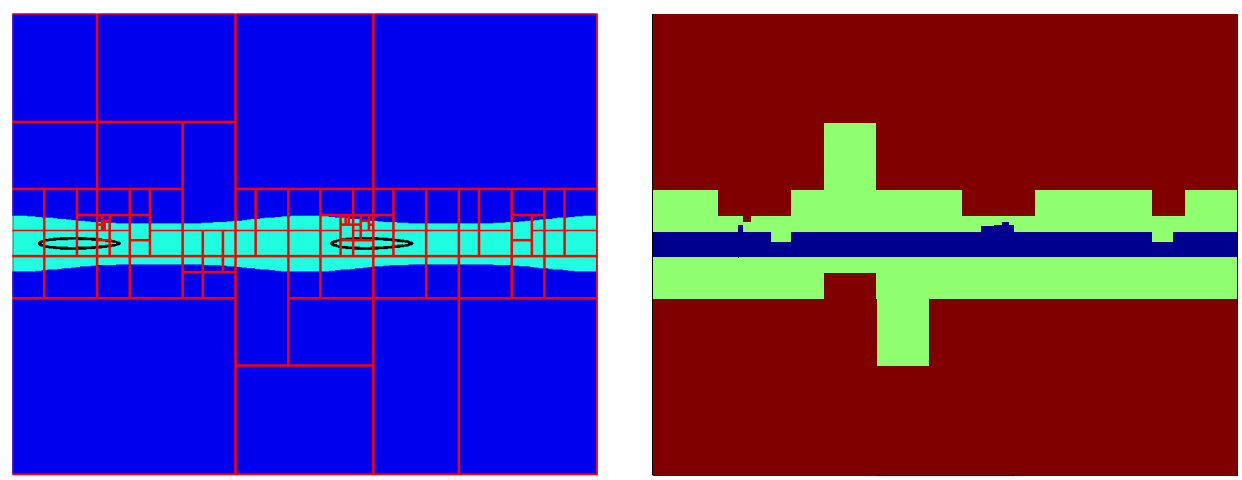

FIG. 15. Isolating block validation for the Hopf bifurcation example (4.5), for $\lambda=-1 / 10$. The two images show the result of the isolating block validation algorithm applied to the isolating block $\mathcal{C}_{1}$. The left image shows the exit set in dark blue, as well as the final adaptive grid produced by the algorithm. In addition, the zero set of the modified tangency test function $w$ is shown in black. The image on the right shows the type of each rectangle in the final grid, color coded according to the number of positive function values of $u$ at the corners of each rectangle, with red, orange, green, light blue, and dark blue corresponding to four, three, two, one, and zero, respectively. In both images, the horizontal axis is for the variable $0 \leq \phi \leq 2 \pi$, while the vertical axis is for $0 \leq \theta \leq \pi$. The images imply that the isolating block $\mathcal{C}_{1}$ contains an isolated invariant set which has the Conley index of a hyperbolic index 1 equilibrium.

the study of three-dimensional flows, we instead consider the system

$$
\begin{aligned}
& \dot{x}=\lambda x+y-x^{3}, \\
& \dot{y}=-x+\lambda y, \\
& \dot{z}=5 z,
\end{aligned}
$$

which is obtained from $[12$, p. 396] by adding an unstable $z$-direction. This system depends on a real parameter $\lambda$, and we are interested in the dynamics of (4.5) as $\lambda$ increases through zero.

One can easily verify that the origin is an equilibrium for (4.5), which is unstable with index 1 for $\lambda<0$, and unstable with index 3 for $\lambda>0$. As $\lambda$ increases through zero, the linearization of (4.5) at the origin has a pair $\lambda \pm i$ of complex eigenvalues which cross the imaginary axis with nonzero speed - hence triggering a Hopf bifurcation. For our application of validated isolating blocks we consider the following two neighborhoods of the origin:

- The set $\mathcal{C}_{1}$ is a ball of radius $3 / 10$ centered at the origin.

- The set $\mathcal{C}_{2}$ is an ellipsoid centered at the origin. Its major axes have lengths 3 , 2 , and 2 , and point in the directions of the unit vectors given in (3.2).

The boundary of either set is parameterized using spherical coordinates as described in Section 3.2. In fact, the example discussed in Sections 3.2 and 3.3 is precisely set $\mathcal{C}_{2}$ defined above, for the ordinary differential equation (4.5) at $\lambda=-1 / 10$.

We first discuss the flow of (4.5) for the case $\lambda=-1 / 10$. Our validation algorithm can be applied to both neighborhoods $\mathcal{C}_{1}$ and $\mathcal{C}_{2}$, and it confirms both as isolating blocks. The corresponding exit set for $\mathcal{C}_{1}$ is shown in dark blue in the left image of Figure 15 , while the exit set for $\mathcal{C}_{2}$ was already presented in the left image of Figure 5 . In both of these images, the zero set of the tangency test function $w$ is shown as black loops, outside of which the function $w$ is strictly positive. Finally, the right images in 

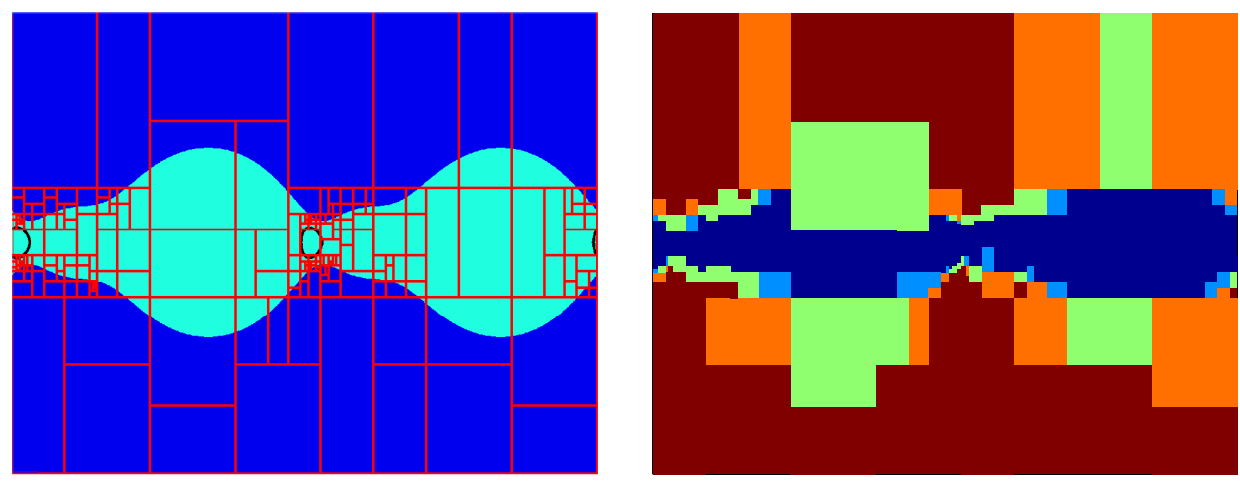

FIG. 16. Isolating block validation for the Hopf bifurcation example (4.5), for $\lambda=1 / 10$. The two images show the result of the isolating block validation algorithm applied to the isolating block $\mathcal{C}_{2}$. The left image shows the exit set in dark blue, as well as the final adaptive grid produced by the algorithm. In addition, the zero set of the modified tangency test function $w$ is shown in black. The image on the right shows the type of each rectangle in the final grid, color coded as in Figure 15 . In both images, the horizontal axis is for the parametrization variable $0 \leq \phi \leq 2 \pi$, while the vertical axis is for $0 \leq \theta \leq \pi$. The images imply that the isolating block $\mathcal{C}_{2}$ contains an isolated invariant set which has the Conley index of a hyperbolic index 1 equilibrium.

Figures 15 and 5 show the type of each rectangle in the final adaptive grid generated by the validation algorithm, color coded according to the number of positive function values of $u$ at the corners of each rectangle, with red, orange, green, light blue, and dark blue corresponding to four, three, two, one, and zero positive function values, respectively. For both isolating blocks $\mathcal{C}_{1}$ and $\mathcal{C}_{2}$ these computations imply that the corresponding homological Conley index is given by

$$
C H_{*}\left(\mathcal{C}_{1}\right) \simeq C H_{*}\left(\mathcal{C}_{2}\right) \simeq(0, \mathbb{Z}, 0,0,0, \ldots),
$$

which is the Conley index of a hyperbolic fixed point with one-dimensional unstable manifold. In fact, for $\lambda<0$ the origin is the unique equilibrium solution of (4.5), and it has index 1.

The situation changes if we turn our attention to the case $\lambda=1 / 10$. Our validation algorithm still can be applied successfully to both $\mathcal{C}_{1}$ and $\mathcal{C}_{2}$. In the case of the set $\mathcal{C}_{1}$, the exit set function $u$ turns out to be strictly positive on $\Omega=[0,2 \pi] \times[0, \pi]$, so the resulting grid contains only one rectangle, and step (IB2) in the validation algorithm completes without any evaluation of the tangency test function $w$. For the isolating block $\mathcal{C}_{2}$, its exit set in shown in dark blue in the left image of Figure 16, the type of each rectangle in the final adaptive grid is shown on the right. In addition, the zero set of the tangency test function $w$ is shown as black loops, outside of which the function $w$ is strictly positive. See also Figure 17 for a rendering of the isolating blocks $\mathcal{C}_{1}$ and $\mathcal{C}_{2}$ at both parameter values $\lambda= \pm 1$, together with their exit sets. These images show that while the homological Conley index of the isolating block $\mathcal{C}_{2}$ remains unchanged, the Conley index of $\mathcal{C}_{1}$ is now given by

$$
C H_{*}\left(\mathcal{C}_{1}\right) \simeq(0,0,0, \mathbb{Z}, 0, \ldots),
$$

which is the expected Conley index of an index 3 unstable equilibrium. Of course, this equilibrium is the origin which is contained in $\mathcal{C}_{1}$. We would like to point out that in contrast to the case $\lambda<0$, the differential equation (4.5) has the additional 

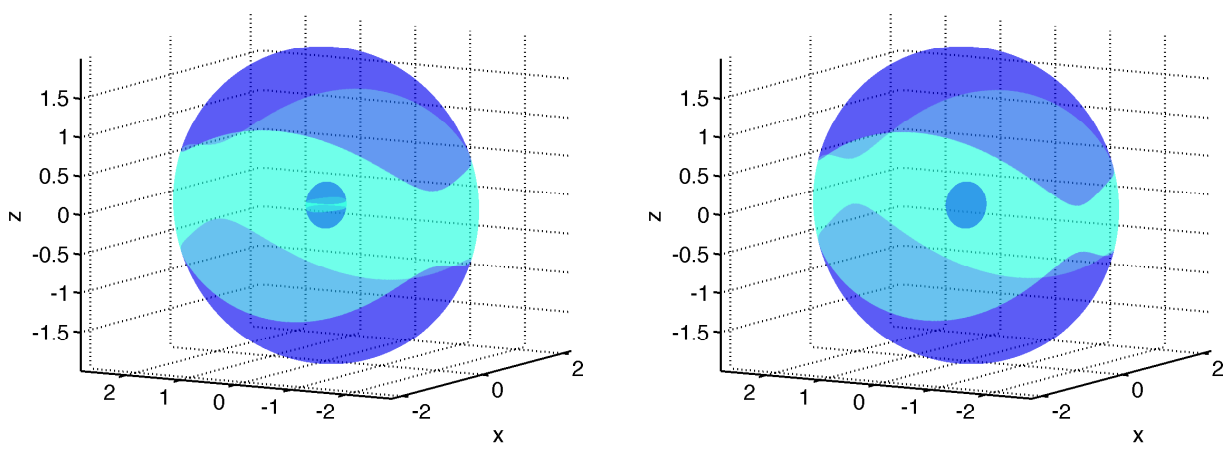

FIG. 17. Visualization of the isolating blocks $\mathcal{C}_{1}$ and $\mathcal{C}_{2}$ and their exit sets in the phase space of (4.5). The left image shows the exit sets for $\lambda=-1 / 10$, the right image for $\lambda=1 / 10$. As described in the text, these images prove that between the two $\lambda$-values a periodic orbit bifurcates from the origin, and that there are connecting orbits from the origin to the periodic orbit for $\lambda=1 / 10$. The periodic orbit is contained in the shell region between the small ball $\mathcal{C}_{1}$ and the large ellipsoid $\mathcal{C}_{2}$.

equilibria

$$
\pm\left(\sqrt{\lambda+\frac{1}{\lambda}}, \sqrt{\frac{1}{\lambda}+\frac{1}{\lambda^{3}}}, 0\right), \quad \text { for } \quad \lambda>0 .
$$

Yet, for our choice $\lambda=1 / 10$ one can easily verify that these additional equilibrium solutions lie outside the isolating blocks $\mathcal{C}_{1}$ and $\mathcal{C}_{2}$.

What does the above Conley index information tell us about the dynamics of (4.5)? From our above discussion of the equilibrium solutions we see that both $\mathcal{C}_{1}$ and $\mathcal{C}_{2}$ only contain one equilibrium, namely the origin - and as $\lambda$ increases from $-1 / 10$ to $1 / 10$ no other equlibria enter these sets. Thus, the index change implies the bifurcation of an invariant set which has to touch $\partial \mathcal{C}_{1}$ for some $\lambda$-value strictly between $-1 / 10$ and $1 / 10$. But we can say even more at $\lambda=1 / 10$. Since the surface $\partial \mathcal{C}_{1}$ consists only of exit points, it is trivial to verify that $\overline{\mathcal{C}_{2} \backslash \mathcal{C}_{1}}$ is an isolating block as well, and that its homological Conley index is given by

$$
C H_{*}\left(\overline{\mathcal{C}_{2} \backslash \mathcal{C}_{1}}\right) \simeq(0, \mathbb{Z}, \mathbb{Z}, 0,0, \ldots) .
$$

This index is non-trivial, and therefore the interior of $\overline{\mathcal{C}_{2} \backslash \mathcal{C}_{1}}$ contains a maximal isolated invariant set $\mathcal{S} \neq \emptyset$. As discussed earlier, the set $\mathcal{S}$ cannot contain any equilibria. Furthermore, due to the form of (4.5), every globally bounded invariant set has to be contained in the $x-y$-plane. But then the Poincaré-Bendixson theorem implies that $\mathcal{S}$ has to contain a periodic orbit. Note that this is consistent with the above Conley index information, as the index is that of an unstable periodic orbit with two-dimensional unstable manifold. Finally, due to

$$
C H_{*}\left(\mathcal{C}_{1}\right) \oplus C H_{*}\left(\overline{\mathcal{C}_{2} \backslash \mathcal{C}_{1}}\right) \not C H_{*}\left(\mathcal{C}_{2}\right),
$$

there has to be at least one connecting orbit from the unstable origin to the isolated invariant set $\mathcal{S}$.

Acknowledgments. This research was supported in part by the Institute for Mathematics and its Applications at the University of Minnesota with funds provided by 
the National Science Foundation. In addition, this work was partially supported by NSF grants DMS-0907818 and DMS-1114923.

\section{REFERENCES}

[1] Erik Boczko, Polygonal Approximation for Flows, PhD thesis, Georgia Institute of Technology, 2002.

[2] Erik Boczko, William D. Kalies, and Konstantin Mischaikow, Polygonal approximation of flows, Topology and its Applications, 154 (2007), pp. 2501-2520.

[3] Gregory S. Cochran, Thomas Wanner, and Pawee Deotko, A Randomized Subdivision Algorithm for Determining the Topology of Nodal Sets, SIAM Journal on Scientific Computing, 35 (2013), pp. B1034-B1054.

[4] Charles Conley, Isolated Invariant Sets and the Morse Index, American Mathematical Society, Providence, R.I., 1978.

[5] Charles Conley and Robert Easton, Isolated invariant sets and isolating blocks, Transactions of the American Mathematical Society, 158 (1971), pp. 35-61.

[6] Sarah Day, William D. Kalies, Konstantin Mischaikow, and Thomas Wanner, Probabilistic and numerical validation of homology computations for nodal domains, Electronic Research Announcements of the American Mathematical Society, 13 (2007), pp. 60-73.

[7] Sarah Day, William D. Kalies, and Thomas Wanner, Verified homology computations for nodal domains, SIAM Journal on Multiscale Modeling \& Simulation, 7 (2009), pp. 16951726.

[8] Pawę DŁotko, Tomasz Kaczynski, Marian Mrozek, and Thomas Wanner, Coreduction homology algorithm for regular CW-complexes, Discrete \& Computational Geometry, 46 (2011), pp. 361-388.

[9] Pawee Deotko, Thomas Stephens, and Thomas Wanner, Randomized adaptive nodal domain validation in arbitrary dimensions, In preparation, (2014).

[10] Manfredo Perdigão do Carmo, Riemannian Geometry, Birkhäuser, 1992.

[11] Dominik EBerlein, Topologische Methoden zur Analyse dynamischer Systeme, PhD thesis, Technische Universität München, 2004.

[12] Dominik Eberlein And Jürgen Scheurle, Algorithmic computation of the Conley index, Journal of Difference Equations and Applications, 12 (2006), pp. 385-398.

[13] Morris W. Hirsch, Differential Topology, vol. 33 of Graduate Texts in Mathematics, Springer, 1994.

[14] Josephus Hulshof And Robertus C. A. M. VAn Der Vorst, Asymptotic behaviour of ground states, Proceedings of the American Mathematical Society, 124 (1996), pp. 2423-2431.

[15] Tomasz Kaczynski, Konstantin Mischaikow, and Marian Mrozek, Computational Homology, vol. 157 of Applied Mathematical Sciences, Springer-Verlag, New York, 2004.

[16] John M. LeE, Riemannian Manifolds, vol. 176 of Graduate Texts in Mathematics, Springer, 1997.

[17] - Introduction to Smooth Manifolds, vol. 218 of Graduate Texts in Mathematics, Springer, second ed., 2013.

[18] Konstantin Mischaikow and Marian Mrozek, Conley index, in Handbook of dynamical systems, Vol. 2, North-Holland, Amsterdam, 2002, pp. 393-460.

[19] Ramon E. Moore, R. Baker Kearfott, and Michael J. Cloud, Introduction to Interval Analysis, SIAM, Philadelphia, 2009.

[20] Siegfried M. Rump, INTLAB - INTerval LABoratory, in Developments in Reliable Computing, Tibor Csendes, ed., Kluwer Academic Publishers, Dordrecht, 1999, pp. 77-104. http://www.ti3.tuhh.de/rump/.

[21] Joel Smoller, Shock Waves and Reaction-Diffusion Equations, Springer-Verlag, New York, second ed., 1994. 